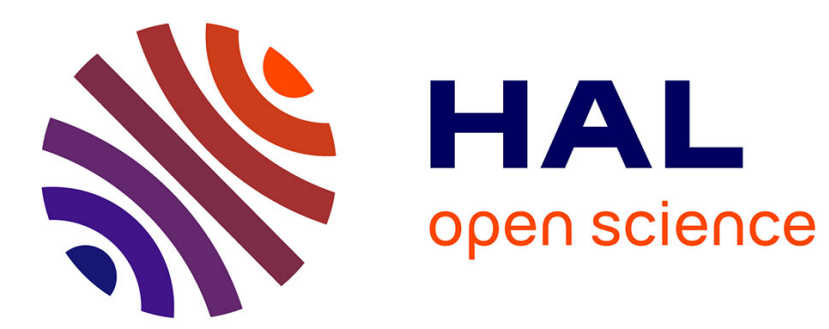

\title{
A CONSERVATIVE AND CONVERGENT SCHEME FOR UNDERCOMPRESSIVE SHOCK WAVES
}

\author{
Christophe Chalons, Patrick Engel, Christian Rohde
}

\section{To cite this version:}

Christophe Chalons, Patrick Engel, Christian Rohde. A CONSERVATIVE AND CONVERGENT SCHEME FOR UNDERCOMPRESSIVE SHOCK WAVES. 2012. hal-00747238

\author{
HAL Id: hal-00747238 \\ https://hal.science/hal-00747238
}

Preprint submitted on 30 Oct 2012

HAL is a multi-disciplinary open access archive for the deposit and dissemination of scientific research documents, whether they are published or not. The documents may come from teaching and research institutions in France or abroad, or from public or private research centers.
L'archive ouverte pluridisciplinaire HAL, est destinée au dépôt et à la diffusion de documents scientifiques de niveau recherche, publiés ou non, émanant des établissements d'enseignement et de recherche français ou étrangers, des laboratoires publics ou privés. 


\title{
A CONSERVATIVE AND CONVERGENT SCHEME FOR UNDERCOMPRESSIVE SHOCK WAVES
}

\author{
CH. CHALONS ${ }^{\dagger}$, P. ENGEL ${ }^{\ddagger}$, AND CH. ROHDE $\ddagger$
}

\begin{abstract}
Undercompressive shock waves arise in numerous physical applications. We propose a class of conservative finite-volume type schemes to approximate weak solutions of conservation laws that contain undercompressive shock waves. We prove the convergence of a subsequence of approximate solutions towards a generalized entropy solution if the mesh width tends to zero. The proof relies on a refined BV compactness analysis, which accounts for the effect of the kinetic relation that drives the undercompressive wave. At the same time we establish a new proof for the existence of solutions to the underlying model. Numerical experiments supplement the analytical results.
\end{abstract}

1. Introduction. Let $f \in \mathcal{C}^{2}(\mathbb{R}), u_{0} \in L^{\infty}(\mathbb{R})$, and $T>0$ be given. We consider solutions $u: \mathbb{R} \times[0, T) \rightarrow \mathbb{R}$ of the scalar conservation law

$$
u_{t}+f(u)_{x}=0 \quad \text { in } \mathbb{R} \times(0, T),
$$

with initial condition

$$
u(\cdot, 0)=u_{0} \quad \text { in } \mathbb{R} .
$$

If the flux is nonlinear, the Cauchy problem (1.1), (1.2) cannot be solved within the class of smooth functions. Therefore, weak solutions, i.e., distributional solutions in $L^{\infty}(\mathbb{R} \times(0, T))$ have to be considered. However, (1.1), (1.2) can have multiple weak solutions. Uniqueness is restored in the class of entropy solutions. That are weak solutions which satisfy the entropy inequality

$$
\eta(u)_{t}+q(u)_{x} \leq 0 \text { for all entropies } \eta \text { in } \mathcal{D}^{\prime}(\mathbb{R} \times[0, T)) .
$$

Thereby, an entropy $\eta \in \mathcal{C}^{2}(\mathbb{R})$ is a convex function and the entropy flux $q \in \mathcal{C}^{2}(\mathbb{R})$ is given through $q^{\prime}=\eta^{\prime} f^{\prime}$. Weak solutions of shock wave type that satisfy the entropy condition (1.3) are necessarily compressive. That means that characteristics impinge on the discontinuity from both sides. We refer to [9] for a comprehensive treatment of the classical scalar well-posedness theory.

In a wide range of applications (cf. $[1,2,6,7,16,29]$ for an incomplete list of examples), discontinuous waves appear that take the form of undercompressive waves. In the scalar case of problem (1.1), undercompressive shock waves are characterized by the fact that characteristics pass through the shock wave. For the precise definition see Section 2 below. Note that this is only possible in the case that the flux in (1.1) has inflection points.

As a matter of fact, undercompressive waves are ruled out by (1.3). In any case, physically relevant weak solutions with undercompressive waves should still be consistent with the second law of thermodynamics. Thus, they are supposed to satisfy

$$
\bar{\eta}(u)_{t}+\bar{q}(u)_{x} \leq 0 \text { for (at least) one entropy } \bar{\eta} \text { in } \mathcal{D}^{\prime}(\mathbb{R} \times[0, T)) .
$$

Obviously condition (1.4) does no ensure unique solvability of the Cauchy problem (1.1), (1.2). Other criteria are required.

\footnotetext{
†Université Paris Diderot Paris 7 \& Laboratoire Jacques-Louis Lions U.M.R. 7598, Boîte courrier 187, 75252 Paris Cedex 05, France. christophe_chalons@ljll.univ-paris-diderot.fr

${ }^{\ddagger}$ Institut für Angewandte Analysis und Numerische Simulation, University of Stuttgart, Pfaffenwaldring 57, D-70569 Stuttgart, Germany. \{patrick.engel, crohde\}@math.uni-stuttgart.de
} 
An approach to guarantee unique solvability is embedding the first order equation (1.1) into an enlarged model of type

$$
u_{t}^{\varepsilon}+f\left(u^{\varepsilon}\right)_{x}=R\left[\varepsilon ; u^{\varepsilon}\right] \quad \text { in } \mathbb{R} \times(0, T) .
$$

Here, $\varepsilon>0$ is a small parameter and $R[\varepsilon ; \cdot]$ some operator with $R[0 ; \cdot] \equiv 0$. In the limit $\varepsilon \rightarrow 0$ the sequence of solutions $\left\{u^{\varepsilon}\right\}_{\varepsilon>0}$ to (1.5), (1.2) selects a unique weak solution for (1.1), (1.2) (provided solutions $u^{\varepsilon}$ exist and converge at all in an appropriate function space). To recover undercompressive waves that satisfy (1.4) in the limit, the operator $R$ has to be chosen such that solutions of (1.5), (1.2) dissipate the entropy $\bar{\eta}$. Examples typically take the form of higher-order differential or integral operators, and can be found in e.g. [2, 8, 12, 26, 29].

Another approach is motivated by the study of configurational forces for phase transformation in solids [1,28]. This ansatz enforces uniqueness by restrictions on undercompressive shock waves through kinetic relations and nucleation criteria. A kinetic relation is an algebraic condition on the traces across an undercompressive shock wave. In view of (1.4) and the application background, kinetic functions are usually chosen to determine exactly the amount of entropy dissipation with respect to $\bar{\eta}$.

For a discussion of both methods and its analytical implications we refer to the book [19].

The numerical approximation of undercompressive waves is a nontrivial issue. On the one hand the exact solutions are neither monotone nor total-variation diminishing [19]. As a consequence, the highly successful finite volume schemes with these properties are ruled out from the beginning. On the other hand, one of the selection mechanisms outlined above has to be accounted for on the discrete level.

Following the idea of embedding, one discretizes first (1.5) and then identifies the role of $\varepsilon$ with the spatial mesh parameter. Thus, one obtains a consistent discretization for (1.1) that mimics the effect of the operator $R$ in (1.5) if the mesh width vanishes. The schemes from the papers $[13,15,20,21,22]$ fall into this class. Up to our knowledge, the proof of convergence for these methods is an open issue.

The other class of schemes relies on the kinetic relation directly. The first of such schemes is the Glimm-type ansatz in [18], see also [5]. Deterministic versions that use an extra tracking of the undercompressive waves have been introduced in $[4,14$, $23,24,30]$. The drawback of all these schemes is the fact that the discrete solution does not conserve the integral of the solution. To overcome this problem, conservative finite-volume type methods have been suggested in [3, 25]. These schemes use a special numerical flux close to undercompressive waves (and standard monotone fluxes away from the wave). From the point of view of error analysis these schemes can usually be proven to converge if the initial data corresponds to a single undercompressive wave that satisfies the given kinetic relation.

In this contribution we introduce a new conservative method. It imposes the kinetic relation directly, and thus belongs to the second class of schemes described above. We prove the convergence towards a generalized entropy solution. The proof applies to solutions which contain a finite number of undercompressive waves but is not restricted to well-prepared initial data as in many previous works.

Our proof does not only yield the weak solution, but does also identify the location of the phase boundary, the associated entropy dissipation, and its characteristic structure. 
In Section 2 we give a rigorous definition of the generalized entropy solutions we are searching for. We introduce the novel method in Section 3. In Section 4 we state the convergence theorem (Theorem 4.4). Section 5 is devoted completely to the proof. The last section contains numerical experiments that illustrate the analytical findings and the future potential of the method.

2. The Mathematical Model. We assume that the flux $f \in \mathcal{C}^{2}(\mathbb{R})$ has a concave-convex shape with inflection point at $u=0$, i.e.,

$$
u f^{\prime \prime}(u)>0 \text { for all } u \in \mathbb{R} \backslash\{0\} \quad \text { and } \quad f^{\prime \prime}(0)=0 .
$$

With this assumption and having in mind the application scenario of phase transformations we define two phases by

$$
\mathcal{Z}_{+}=\{u>0\} \quad \text { and } \quad \mathcal{Z}_{-}=\{u<0\} .
$$

By a phase boundary we mean a function $u=u(x, t)$ of shock wave type, i.e.,

$$
u(x, t)=\left\{\begin{array}{lll}
u_{-} & : & x-\bar{a} t<0 \\
u_{+} & : & x-\bar{a} t>0
\end{array} \quad \bar{a}=\bar{a}\left(u_{-}, u_{+}\right):=\frac{f\left(u_{-}\right)-f\left(u_{+}\right)}{u_{-}-u_{+}},\right.
$$

such that either $u_{ \pm} \in \mathcal{Z}_{ \pm}$or $u_{ \pm} \in \mathcal{Z}_{\mp}$ holds. The choice of $\bar{a}$ guarantees the validity of the Rankine-Hugoniot conditions. Phase boundaries can be of compressive/Laxian type if

$$
f^{\prime}\left(u_{+}\right) \leq \bar{a} \leq f^{\prime}\left(u_{-}\right)
$$

holds, or of undercompressive type if either

$$
f^{\prime}\left(u_{+}\right)<\bar{a}, \bar{a}>f^{\prime}\left(u_{-}\right) \quad \text { or } \quad f^{\prime}\left(u_{+}\right)>\bar{a}, \bar{a}<f^{\prime}\left(u_{-}\right)
$$

hold. Note that by (2.3) also the (one-sided) characteristic case is included for the sake of simplicity.

We are in particular interested in weak solutions of (1.1) that contain undercompressive phase boundaries. For the initial data we assume that $u_{0} \in\left(\mathrm{BV} \cap L^{1}\right)(\mathbb{R})$ and

$$
x u_{0}(x)<0
$$

holds for almost all $x \in \mathbb{R}$. The property (2.5) implies that exactly one phase change at $x=0$ is prescribed by $u_{0}$.

The extension of the analysis of this paper to initial data with (finitely) many phase boundaries is possible but would lead to a much more heavy notational overhead. In view of phase transformation applications the assumption (2.1) on the flux function appears to be a generic situation [1]. The treatment of the interesting case of flux functions with multiple inflection points and thus problems with multiple phases is out of the scope of this paper.

As the next step, we introduce a generalized notion of entropy solution for weak solutions that contain undercompressive waves. Let us recall that a weak solution of (1.1) is a function $u \in L^{\infty}(\mathbb{R} \times(0, T))$ such that

$$
\int_{\mathbb{R}_{\mathbb{R}^{+}}} u \psi_{t}+f(u) \psi_{x} \mathrm{~d} x \mathrm{~d} t=-\int_{\mathbb{R}} \psi(x, 0) u_{0}(x) \mathrm{d} x
$$


holds for all $\psi \in C_{0}^{1}(\mathbb{R} \times[0, T))$. Rewriting (1.4) leads to

$$
\int_{\mathbb{R} \times \mathbb{R}^{+}} \bar{\eta}(u) \psi_{t}+\bar{q}(u) \psi_{x} \mathrm{~d} x \mathrm{~d} t \geq-\int_{\mathbb{R}} \psi(x, 0) \bar{\eta}\left(u_{0}(x)\right) \mathrm{d} x
$$

for all non-negative functions $\psi \in C_{0}^{1}(\mathbb{R} \times[0, T))$.

In recent years several well-posedness theories for weak solutions have been developed that allow for undercompressive waves [19]. We detail here the concept of a kinetic relation.

Definition 2.1 (Kinetic Relation). A function $\varphi: \mathcal{Z}_{+} \rightarrow \mathcal{Z}_{-}$is called kinetic relation if

$$
\begin{gathered}
\exists L>0:\left|\varphi(u)-\varphi\left(u^{\prime}\right)\right| \leq L\left|u-u^{\prime}\right| \forall u, u^{\prime} \in \mathcal{Z}_{+}, \\
\varphi \text { is decreasing, } \\
\varphi^{\sharp}(u) \geq \varphi(u) \quad \text { and } \quad \varphi(0)=0
\end{gathered}
$$

hold. In (2.8c) the function $\varphi^{\sharp}: \mathcal{Z}_{+} \rightarrow \mathcal{Z}_{-}$is defined by

$$
\bar{a}\left(u, \varphi^{\sharp}(u)\right)=\bar{a}(u, \varphi(u)) \quad \text { and } \quad \varphi^{\sharp}(u) \neq \varphi(u) .
$$

According to (2.1) the function $\varphi^{\sharp}$ is well-defined.

A kinetic relation $\varphi: \mathcal{Z}_{+} \rightarrow \mathcal{Z}_{-}$is called compatible with an entropy $\bar{\eta} \in \mathcal{C}^{2}(\mathbb{R})$ if

$$
\bar{a}(u, \varphi(u))(\bar{\eta}(u)-\bar{\eta}(\varphi(u)))-(\bar{q}(u)-\bar{q}(\varphi(u))) \leq 0
$$

holds for all $u \in \mathcal{Z}_{+}$, with $\bar{a}$ as in (2.2). To illustrate the interplay of kinetic relations and the notion of entropy compatibility consider the following example that will also be used later in Section 6 .

EXAMPLE 2.2. Let $f(u)=\frac{1}{3} u^{3}+c u$ with $c \in \mathbb{R}$ and the kinetic relation $\varphi(u)=$ $-\kappa u$ with $\kappa \in\left[\frac{1}{2}, 1\right]$ be given. Consider the entropy pairs $(\eta, q)$ given by

$$
\eta(u)=\frac{1}{r} u^{r} \quad \text { and } \quad q(u)=\frac{1}{r+2} u^{r+2}+\frac{c}{r} u^{r}, \quad \frac{r}{2} \in \mathbb{N} .
$$

The left-hand side of (2.10) is computed as

$$
\frac{2(r-1)\left(\kappa^{r+2}-1\right)+(r+2)\left(\kappa^{r+1}-\kappa^{r}+\kappa^{2}-\kappa\right)}{3 r(r+2)} u^{r+2} \leq 0,
$$

thus $\varphi$ is compatible with all entropies from (2.11).

In the following we will construct an entropy which is not compatible with $\varphi$. We choose for $k \in \mathbb{R}$ the Kruzkov entropy $\eta(u)=|u-k|$ with entropy flux $q(u)=\operatorname{sign}(u-$ $k)(f(u)-f(k))$. Fix $u \in \mathcal{Z}_{+}$and take $k \in[\varphi(u), u]$, then (2.10) is equivalent to

$$
t(k)=\bar{a}(u, \varphi(u))(u+\varphi(u)-2 k)-(f(u)+f(\varphi(u))-2 f(k)) \leq 0 .
$$

If we maximize $t(k)$ for $k \in[\varphi(u), u]$ we find the maximum at $k^{*}=-\frac{1}{3} \sqrt{3 \kappa^{2}-3 \kappa+3} u$ and $t\left(k^{*}\right)>0$ for $\kappa \in\left[\frac{1}{2}, 1\right]$. In the sequel we search for functions in the space

$$
V:=\left\{v \in C^{0}\left([0, T] ; L^{1}(\mathbb{R})\right) \mid v(\cdot, t) \in \mathrm{BV}(\mathbb{R}), \forall t \in[0, T]\right\} .
$$


We can now present our generalized notion of entropy solutions.

Definition $2.3\left((\bar{\eta}, \varphi)\right.$-solution). Let an entropy $\bar{\eta} \in \mathcal{C}^{2}(\mathbb{R})$ and a kinetic relation $\varphi$ be given such that $\varphi$ is compatible with $\bar{\eta}$.

$A$ weak solution $u \in V$ of (1.1) together with $p \in C^{0}(0, T)$ is called an $(\bar{\eta}, \varphi)$-solution of (1.1) if it satisfies the following properties:

1. The function u fulfills the entropy condition (2.7) for $\bar{\eta}$.

2. $p$ is differentiable for almost all $t \in(0, T)$ and fulfills there

$$
p_{t}(t)=\bar{a}\left(u_{-}(t), u_{+}(t)\right) .
$$

3. For almost all $t \in(0, T)$ we have

$$
u_{+}(t)=\varphi\left(u_{-}(t)\right) \quad \text { or } \quad u_{+}(t) \geq \varphi^{\sharp}\left(u_{-}(t)\right) .
$$

In (2.13) and (2.14) the function $u_{ \pm}:[0, T) \rightarrow \mathbb{R}$ is defined by

$$
u_{ \pm}(t):=\lim _{\varepsilon \rightarrow 0} u(p(t) \pm \varepsilon, t) .
$$

REMARK 2.4.

(i) Our notion of solution should be understood as a free-boundary formulation for phase boundaries. The function $p$ tracks the position of the phase boundary.

(ii) Undercompressive waves cannot satisfy (2.7) for all entropies. Nevertheless in applications with undercompressive waves some physical energy function is naturally dissipated. In fact kinetic relations occur in applications as dissipation rates for the (negative of the) physical entropy. This motivates the definition of entropy compatible kinetic relations and the first condition in Definition 2.3.

(iii) We recall that phase boundaries can be either Laxian or undercompressive waves. As we will see later in Example 6.4 a phase boundary can change its type which has to be reflected in the formulation of the weak solution: the first condition in (2.14) identifies the discontinuity across $p$ as an undercompressive case, the second one as Laxian wave (see [19]).

(iv) The limits in (2.15) are well-defined, since $u(\cdot, t) \in \mathrm{BV}(\mathbb{R})$ for all $t \in[0, T]$.

(v) In view of assumption (2.5) we will have $u_{-}(t) \in \mathcal{Z}_{+}$in (2.14). In the general case the inequality in (2.14) should be written as

$$
u_{-}(t) u_{+}(t) \geq u_{-}(t) \varphi^{\sharp}\left(u_{-}(t)\right) .
$$

We believe that the notion of weak solution from Definition 2.3 can be used to prove the well-posedness of the initial value problem (1.1). Rigorous proofs for existence and uniqueness can be found in [19]. Let us discuss here the Riemann problem for the sake of illustration. Consider for $u_{L}>0$ and $u_{R} \in \mathbb{R}$ the Riemann initial data

$$
u_{0}(x)= \begin{cases}u_{L} & \text { for } x<0, \\ u_{R} & \text { for } x>0 .\end{cases}
$$

The cases $u_{R} \geq u_{L}$ and $u_{R} \in\left[0, u_{L}\right)$ correspond to one-phase initial data. They are solved by a single rarefaction wave and a single Laxian shock wave, respectively. We concentrate on two-phase initial datum (cf. (2.5)). 
EXAMPLE 2.5. Recalling the results from [19] we know that solutions of the Riemann problem (1.1), (2.16) have the following structure for a given kinetic relation $\varphi: \mathcal{Z}_{+} \rightarrow \mathcal{Z}_{-}:$

(i) $u_{R} \in\left[\varphi^{\sharp}\left(u_{L}\right), 0\right)$ : The solution

$$
u(x, t)= \begin{cases}u_{L} & \text { for } \frac{x}{t} \leq \bar{a}\left(u_{L}, u_{R}\right) \\ u_{R} & \text { for } \bar{a}\left(u_{L}, u_{R}\right) \leq \frac{x}{t}\end{cases}
$$

is a Laxian wave.

(ii) $u_{R} \in\left[\varphi\left(u_{L}\right), \varphi^{\sharp}\left(u_{L}\right)\right)$ : The solution

$$
u(x, t)= \begin{cases}u_{L} & \text { for } \frac{x}{t} \leq \bar{a}\left(u_{L}, \varphi\left(u_{L}\right)\right) \\ \varphi\left(u_{L}\right) & \text { for } \bar{a}\left(u_{L}, \varphi\left(u_{L}\right)\right) \leq \frac{x}{t} \leq \bar{a}\left(\varphi\left(u_{L}\right), u_{R}\right) \\ u_{R} & \text { for } \bar{a}\left(\varphi\left(u_{L}\right), u_{R}\right) \leq \frac{x}{t}\end{cases}
$$

is an undercompressive phase boundary followed by a shock wave.

(iii) $u_{R}<\varphi\left(u_{L}\right)$ : The solution

$$
u(x, t)= \begin{cases}u_{L} & \text { for } \frac{x}{t} \leq \bar{a}\left(u_{L}, \varphi\left(u_{L}\right)\right) \\ \varphi\left(u_{L}\right) & \text { for } \bar{a}\left(u_{L}, \varphi\left(u_{L}\right)\right) \leq \frac{x}{t} \leq f^{\prime}\left(\varphi\left(u_{L}\right)\right) \\ \left(\left.f^{\prime}\right|_{\mathcal{Z}_{-}}\right)^{-1}\left(\frac{x}{t}\right) & \text { for } f^{\prime}\left(\varphi\left(u_{L}\right) \leq \frac{x}{t} \leq f^{\prime}\left(u_{R}\right)\right. \\ u_{R} & \text { for } f^{\prime}\left(u_{R}\right) \leq \frac{x}{t}\end{cases}
$$

is an undercompressive phase boundary followed a rarefaction wave.

Note that the solution from (ii) is neither monotone nor total variation diminishing. Both properties hold for classical entropy solutions [9]. It is easy to check that the function $u$ in (i), (ii), and (iii) is an $(\eta, \varphi)$-solution for all entropies (2.11).

3. The Numerical Method. In this section we propose a numerical method to approximate $(\bar{\eta}, \varphi)$-solutions in the sense of Definition 2.3. The approximation of $(\bar{\eta}, \varphi)$-solutions requires a very accurate flux balancing close to the undercompressive waves. In particular the numerical method must detect two-wave structures as in cases (ii) and (iii) in Example 2.5. Standard finite volume schemes fail in these situations. Therefore, the new explicit time-stepping method uses a background grid in the regions away from the phase boundary. At the phase boundary we cut the space-time cells into two smaller cells according to its exact position. As this might lead to extremely small cells and corresponding vanishing time steps, the cutted cells are merged with the closest spatial background grid cell in the same phase. Then we use this time-space calculation grid to perform an explicit time stepping with exact flux balancing at the interface and standard fluxes away from the phase boundary. Finally, for the new time step, the results are projected to the spatial part of the background grid. The approach is in the spirit of moving mesh methods [27]. We give some illustration in Figure 3.1. The background grid is plotted with dotted lines, the space calculation grid with solid lines. Note that the cell edges of the calculation grid might vary during a single time step.

For the formal presentation of the method in Algorithm 3.1 below, consider a uniform (for the sake of simplicity) background grid on $\mathbb{R} \times[0, T)$ with mesh parameter 


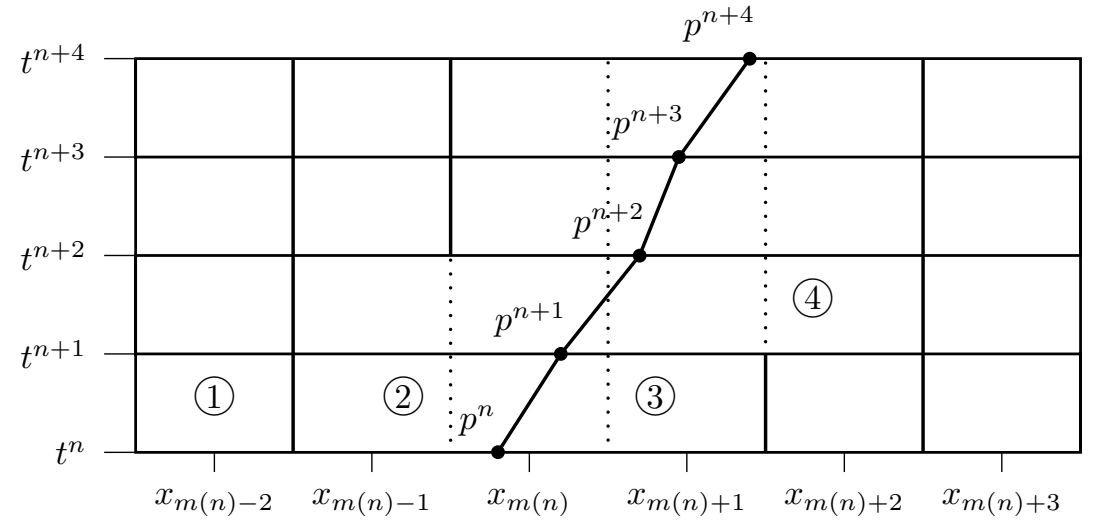

FIG. 3.1. Background and calculation grid for four different geometric configurations (1) to (4). A possible fifth case is described as the mirrored case to (4).

$h>0$. It consists for spatial cells $I_{j}:=\left[x_{j-\frac{1}{2}}, x_{j+\frac{1}{2}}\right)$ of elements $I_{j} \times\left[t^{n}, t^{n+1}\right)$ with

$$
x_{j+\frac{1}{2}}:=\left(j+\frac{1}{2}\right) h \quad(j \in \mathbb{Z}) \text { and } 0=t^{0}<t^{1}<\cdots<t^{N}:=T \text { for some } N \in \mathbb{N} .
$$

The time steps will be related to the spatial mesh parameter by a CFL-like condition. The spatial grid midpoints are given by $x_{j}:=j h$.

The discrete position of the phase boundary at time $t^{n}$ is denoted as $p^{n} \in \mathbb{R}$. We represent $p^{n}$ uniquely with numbers $m(n) \in \mathbb{Z}$ and $\alpha^{n} \in[0,1)$ by the formula

$$
p^{n}=\left(m(n)+\alpha^{n}-\frac{1}{2}\right) h .
$$

Thus $m(n)$ denotes the index of the spatial cell, where the discrete phase boundary is located, i.e., $p^{n} \in I_{m(n)}$. At $t=0$ we have $p^{0}=0$ and therefore $m(0)=0$ and $\alpha^{0}=\frac{1}{2}$. Furthermore, we use the following notations: We define $\Delta m(n):=m(n+1)-m(n)$ and the set $J(n)$ by

$$
J(n):=\mathbb{Z} \backslash\{m(n)-1, m(n), m(n)+1\} .
$$

We write $\Delta m^{+}(n):=\max (0, \Delta m(n))$ and $\Delta m^{-}(n):=-\min (0, \Delta m(n))$. Last, we introduce the indices $m^{-}(n)=m(n)-\Delta m^{-}(n)$ and $m^{+}(n)=m(n)+\Delta m^{+}(n)$.

We define the initial values for $j \in J(0)$ by integral averages, i.e.,

$$
u_{j}^{0}:=\frac{1}{h} \int_{x_{j-\frac{1}{2}}}^{x_{j+\frac{1}{2}}} u_{0}(x) \mathrm{d} x
$$

and $u_{1}^{0}, u_{-1}^{0}$ by

$$
u_{1}^{0}:=\frac{2}{3 h} \int_{0}^{\frac{3}{2} h} u_{0}(x) \mathrm{d} x, \quad u_{-1}^{0}:=\frac{2}{3 h} \int_{-\frac{3}{2} h}^{0} u_{0}(x) \mathrm{d} x .
$$

Note that $u_{0}^{0}$ is not initialized. Furthermore, we ensure that $u_{j}^{0} \in \mathcal{Z}_{ \pm} \Leftrightarrow j \in \mathbb{Z}_{\mp}$.

Let $F: \mathbb{R}^{2} \rightarrow \mathbb{R}$ be a numerical flux, i.e., $F(u, u)=f(u)$ for $u \in \mathbb{R}$. Finally we select an entropy $\bar{\eta} \in C^{2}$ and a kinetic relation $\varphi: \mathcal{Z}_{+} \rightarrow \mathcal{Z}_{-}$which is compatible with $\bar{\eta}$. 
Algorithm 3.1. Let $\left\{u_{j}^{n}\right\}_{j}$ and $p^{n}$ be given for $n \in \mathbb{N}_{0}$.

Then $\left\{u_{j}^{n+1}\right\}_{j}$ and $p^{n+1}$ are calculated by the following procedure.

1. Consider the $(\bar{\eta}, \varphi)$-solution of (1.1), (1.2), (2.16) with $u_{L}=u_{m(n)-1}^{n}$ and $u_{R}=u_{m(n)+1}^{n}$. Define the velocity $s^{n}$ of the phase boundary by

$$
s^{n}:= \begin{cases}\bar{a}\left(\varphi\left(u_{m(n)-1}^{n}\right), u_{m(n)-1}^{n}\right) & \text { if } u_{m(n)+1}^{n}<\varphi^{\sharp}\left(u_{m(n)-1}^{n}\right), \\ \bar{a}\left(u_{m(n)+1}^{n}, u_{m(n)-1}^{n}\right) & \text { if } u_{m(n)+1}^{n} \geq \varphi^{\sharp}\left(u_{m(n)-1}^{n}\right),\end{cases}
$$

and the numerical phase boundary flux $h^{n}$ by

$$
h^{n}:=f\left(u_{m(n)-1}^{n}\right)-s^{n} u_{m(n)-1}^{n} .
$$

2. Define the time step $\Delta t$ through

$$
\Delta t:=\frac{h}{8 \hat{C}} .
$$

For the definition of the constant $\hat{C}>0$ see Assumption 4.2 below.

3. Calculate $m(n+1) \in \mathbb{Z}$ and $\alpha^{n+1} \in[0,1)$ from $\alpha^{n}$ and $s^{n}$ such that

$$
m(n+1)+\alpha^{n+1}=m(n)+\alpha^{n}+\frac{\Delta t}{h} s^{n}
$$

holds.

4. Calculate $\left\{u_{j}^{n+1}\right\}_{j}$ by the following formulas. For $j \in J(n) \cap J(n+1)$ (type (1) in Figure 3.1) we use

$$
u_{j}^{n+1}:=u_{j}^{n}-\frac{\Delta t}{h}\left(f_{j+\frac{1}{2}}^{n}-f_{j-\frac{1}{2}}^{n}\right), \quad f_{j+\frac{1}{2}}^{n}=F\left(u_{j}^{n}, u_{j+1}^{n}\right) .
$$

We give the formulas for the other cells for the different cases $\Delta m(n) \in$ $\{-1,0,1\}$ separately: For $\Delta m(n)=0$ (type (2) and (3) in Figure 3.1) we have

$$
\begin{aligned}
& u_{m(n+1)-1}^{n+1}:=\frac{\left(1+\alpha^{n}\right) u_{m(n)-1}^{n}-\frac{\Delta t}{h}\left(h^{n}-f_{m(n)-\frac{3}{2}}^{n}\right)}{1+\alpha^{n+1}}, \\
& u_{m(n+1)+1}^{n+1}:=\frac{\left(2-\alpha^{n}\right) u_{m(n)+1}^{n}-\frac{\Delta t}{h}\left(f_{m(n)+\frac{3}{2}}^{n}-h^{n}\right)}{2-\alpha^{n+1}} .
\end{aligned}
$$

For $\Delta m(n)=1$ (type (2) and (4) in Figure 3.1) we have

$$
\begin{aligned}
& u_{m(n+1)-2}^{n+1}:=u_{m(n+1)-1}^{n+1}:=\frac{\left(1+\alpha^{n}\right) u_{m(n)-1}^{n}-\frac{\Delta t}{h}\left(h^{n}-f_{m(n)-\frac{3}{2}}^{n}\right)}{2+\alpha^{n+1}}, \\
& u_{m(n+1)+1}^{n+1}:=\frac{\left(2-\alpha^{n}\right) u_{m(n)+1}^{n}+u_{m(n)+2}^{n}-\frac{\Delta t}{h}\left(f_{m(n)+\frac{5}{2}}^{n}-h^{n}\right)}{2-\alpha^{n+1}} .
\end{aligned}
$$

For $\Delta m(n)=-1$ (type (5) and (3) in Figure 3.1) we get

$$
\begin{aligned}
& u_{m(n+1)-1}^{n+1}:=\frac{\left(1+\alpha^{n}\right) u_{m(n)-1}^{n}+u_{m(n)-2}^{n}-\frac{\Delta t}{h}\left(h^{n}-f_{m(n)-\frac{5}{2}}^{n}\right)}{1+\alpha^{n+1}}, \\
& u_{m(n+1)+1}^{n+1}:=u_{m(n+1)+2}^{n+1}:=\frac{\left(2-\alpha^{n}\right) u_{m(n)+1}^{n}-\frac{\Delta t}{h}\left(f_{m(n)+\frac{3}{2}}^{n}-h^{n}\right)}{3-\alpha^{n+1}} .
\end{aligned}
$$


The three expressions (3.8), (3.9), and (3.10) can be summarized in the compact form

$$
\left.\begin{array}{l}
u_{m(n+1)-1}^{n+1} \\
u_{m^{-}(n)-1}^{n+1}
\end{array}\right\}:=\frac{\left(1+\alpha^{n}\right) u_{m(n)-1}^{n}+\Delta m^{-}(n) u_{m(n)-2}^{n}-\frac{\Delta t}{h}\left(h^{n}-f_{m^{-}(n)-\frac{3}{2}}^{n}\right)}{1+\Delta m^{+}(n)+\alpha^{n+1}}
$$

and

$$
\left.\begin{array}{l}
u_{m(n+1)+1}^{n+1} \\
u_{m^{+}(n)+1}^{n+1}
\end{array}\right\}:=\frac{\left(2-\alpha^{n}\right) u_{m(n)+1}^{n}+\Delta m^{+}(n) u_{m(n)+2}^{n}-\frac{\Delta t}{h}\left(f_{m+(n)+\frac{3}{2}}^{n}-h^{n}\right)}{2+\Delta m^{-}(n)-\alpha^{n+1}} .
$$

Finally, let us define the approximate $(\bar{\eta}, \varphi)$-solution $u_{h}: \mathbb{R} \times[0, T] \rightarrow \mathbb{R}$ for $x \in I_{j}$ and $t \in\left[t^{n}, t^{n+1}\right)$ through

$$
u_{h}(x, t)= \begin{cases}u_{j}^{n} & \text { for } j \in J(n), \\ u_{m(n)-1}^{n} & \text { for } j \notin J(n) \text { and } x<p^{n}+s^{n}\left(t-t^{n}\right), \\ u_{m(n)+1}^{n} & \text { for } j \notin J(n) \text { and } x \geq p^{n}+s^{n}\left(t-t^{n}\right) .\end{cases}
$$

The symbol $u_{h}^{n}$ denotes $u_{h}\left(\cdot, t^{n}\right)$. As the first simple but striking property of Algorithm 3.1 we get

Proposition 3.2. Algorithm 3.1 is conservative, i.e., for all $n \in \mathbb{N}$

$$
\int_{\mathbb{R}} u_{h}\left(x, t^{n}\right) \mathrm{d} x=\int_{\mathbb{R}} u_{0}(x) \mathrm{d} x .
$$

Proof. From (3.1), (3.6), (3.7), (3.11), and (3.12) we get

$$
\begin{aligned}
& \int_{\mathbb{R}} u_{h}\left(x, t^{n+1}\right) \mathrm{d} x \\
&= h\left(\sum_{j \in J(n+1)} u_{j}^{n+1}+\left(1+\alpha^{n+1}\right) u_{m(n+1)-1}^{n+1}+\left(2-\alpha^{n+1}\right) u_{m(n+1)+1}^{n+1}\right) \\
&= h\left(\sum_{j \in J(n) \cap J(n+1)} u_{j}^{n+1}+\left(1+\Delta m^{+}(n)+\alpha^{n+1}\right) u_{m(n+1)-1}^{n+1}\right. \\
&\left.+\left(2+\Delta m^{-}(n)-\alpha^{n+1}\right) u_{m(n+1)+1}^{n+1}\right) h\left(\sum_{j \in J(n) \cap J(n+1)} u_{j}^{n}+\Delta m^{-}(n) u_{m(n)-2}^{n}+\left(1+\alpha^{n}\right) u_{m(n)-1}^{n}\right. \\
&\left.+\left(2-\alpha^{n}\right) u_{m(n)+1}^{n}+\Delta m^{+}(n) u_{m(n)+2}^{n}\right) \\
&= h\left(\sum_{j \in J(n)} u_{j}^{n}+\left(1+\alpha^{n}\right) u_{m(n)-1}^{n}+\left(2-\alpha^{n}\right) u_{m(n)+1}^{n}\right)
\end{aligned}
$$




$$
=\int_{\mathbb{R}} u_{h}\left(x, t^{n}\right) \mathrm{d} x,
$$

which implies the statement by induction.

REMARK 3.3. Thanks to the time step restriction (3.5) we have $\Delta m(n) \in$ $\{-1,0,1\}$. Therefore, $\Delta m^{+}(n), \Delta m^{-}(n) \in\{0,1\}$. We interpret $\Delta m^{+}(n)\left(\Delta m^{-}(n)\right)$ as a trigger telling whether the phase boundary moves one cell to the right (left) or not. Thus the following equalities hold:

$$
\begin{aligned}
\Delta m^{+}(n) u_{m(n)+2}^{n} & =\Delta m^{+}(n) u_{m^{+}(n)+1}^{n} \\
\left(1-\Delta m^{+}(n)\right) u_{m(n)+1}^{n} & =\left(1-\Delta m^{+}(n)\right) u_{m^{+}(n)+1}^{n} .
\end{aligned}
$$

4. The Main Theorem. In this section we state the main result. The proof will be given in the subsequent Section 5. We summarize the assumptions on the model and the scheme.

Assumption 4.1 (Assumptions on the Model). Let $f \in C^{2}(\mathbb{R})$ with (2.1) and $\bar{\eta} \in C^{2}(\mathbb{R})$ be a fixed entropy. Let $\varphi: \mathcal{Z}_{+} \rightarrow \mathcal{Z}_{-}$be a kinetic relation in the sense of Definition 2.1 that is compatible with $\bar{\eta}$. Let $u_{0} \in\left(L^{1} \cap \mathrm{BV}\right)(\mathbb{R})$ with $(2.5)$.

Assumption 4.2 (Assumptions on the Scheme). Let $M:=\max (1, L)\left\|u_{0}\right\|_{L^{\infty}(\mathbb{R})}$. Let $F \in C^{1}\left(\mathbb{R}^{2}, \mathbb{R}\right)$ be a numerical flux consistent with $f$, which is monotone and locally Lipschitz-continuous, i.e.,

$$
\begin{gathered}
F(u, u)=f(u), \\
F(\cdot, u) \text { is increasing, } \quad F(u, \cdot) \text { is decreasing, } \\
\left|F(u, v)-F\left(u^{\prime}, v^{\prime}\right)\right| \leq \hat{C}\left(\left|u-u^{\prime}\right|+\left|v-v^{\prime}\right|\right)
\end{gathered}
$$

for all $u, u^{\prime}, v, v^{\prime} \in[-M, M]$. Here $\hat{C}$ is the local Lipschitz constant of $F$ on the domain $[-M, M]^{2}$. Let $Q \in C^{1}\left(\mathbb{R}^{2}, \mathbb{R}\right)$ be a numerical entropy flux function, which is consistent to $\bar{q}$, local Lipschitz-continuous, and compatible to $F$, i.e.,

$$
\begin{gathered}
Q(u, u)=\bar{q}(u), \\
\exists C=C(M):\left|F(u, v)-F\left(u^{\prime}, v^{\prime}\right)\right| \leq C(M)\left(\left|u-u^{\prime}\right|+\left|v-v^{\prime}\right|\right), \\
\partial_{2} Q(u, v)=\bar{\eta}^{\prime}(v) \partial_{2} F(u, v)
\end{gathered}
$$

for all $u, v, u^{\prime}, v^{\prime} \in[-M, M]$.

REMARK 4.3. Let us mention that there are consistent, monotone, and locally Lipschitz-continuous numerical fluxes that allow the construction of a corresponding numerical entropy flux with (4.2). Examples are provided by the Lax-Friedrichs and the Enquist-Osher flux [17].

ThEOREM 4.4 (Convergence and Existence). Let Assumptions 4.1 and 4.2 be fulfilled. Consider for $h>0$ the sequence $\left\{u_{h}\right\}_{h>0}$ of approximate solutions given by Algorithm 3.1. Then we have:

- There exists a subsequence $\left\{u_{h_{n}}\right\}_{n \in \mathbb{N}}$ of $\left\{u_{h}\right\}_{h>0}$ and $u \in V$ such that

$$
\lim _{n \rightarrow \infty}\left\|u_{h_{n}}-u\right\|_{L^{1}}=0 .
$$

- The limit $u$ is a $(\bar{\eta}, \varphi)$-solution of (1.1) in the sense of Definition 2.3. 
If we additionally assume that there exists a unique solution, then the statement is also true for the complete sequence.

Proof. The proof is the combination of the Lemmata 5.3, 5.4, and 5.6, Helly's Theorem, Theorem 5.8, and Theorem 5.13 (see below).

\section{Proof of Theorem 4.4.}

5.1. Preliminary Note. Lemma 5.1 (Properties of $\alpha^{n+1}$ and $s^{n}$ ). Under the assumptions of Theorem 4.4, we have for $n=0, \ldots, N-1$ the inequalities

$$
\begin{array}{ccc}
\Delta m(n)=1 & \Longrightarrow & \alpha^{n+1} \leq \frac{1}{4}, \\
\Delta m(n)=-1 & \Longrightarrow & \alpha^{n+1} \geq \frac{3}{4} .
\end{array}
$$

Furthermore, if $u_{m(n)+1}^{n}<\varphi^{\sharp}\left(u_{m(n)-1}^{n}\right)$, then we have for all $\xi \in\left(-\infty, \varphi^{\sharp}\left(u_{m(n)-1}^{n}\right)\right)$

$$
f^{\prime}(\xi)-s^{n} \geq 0
$$

Proof. From (3.5), (3.6), and $\Delta m(n)=m(n+1)-m(n)$ we get

$$
\alpha^{n+1}=\alpha^{n}+\frac{\Delta t}{h} s^{n}-\Delta m(n) \begin{cases}\leq \frac{\Delta t}{h} \hat{C} \leq \frac{1}{4} & \text { for } \Delta m(n)=1 \\ \geq 1-\frac{\Delta t}{h} \hat{C} \geq \frac{3}{4} & \text { for } \Delta m(n)=-1 .\end{cases}
$$

Note that $\alpha^{n} \in[0,1)$ for all $n \geq 0$.

The inequality (5.2) follows directly from (2.1).

In this section we will prove Theorem 4.4 step by step. Note that we will extract several times subsequence of $\left\{u_{h}\right\}_{h>0}$. All subsequences are also denoted by $\left\{u_{h}\right\}_{h>0}$ to avoid an unnecessary notation overload.

5.2. Reformulation of the Scheme in Harten Form. As a first step, we rewrite Algorithm 3.1 in a Harten-like form (see [11]), which enables us to apply BV compactness theory. We get for $j \in J(n) \cap J(n+1)$ the formula

$$
u_{j}^{n+1}=u_{j}^{n}+\frac{\Delta t}{h} C_{j+\frac{1}{2}}^{n}\left(u_{j+1}^{n}-u_{j}^{n}\right)-\frac{\Delta t}{h} D_{j-\frac{1}{2}}^{n}\left(u_{j}^{n}-u_{j-1}^{n}\right)
$$

with

$$
C_{j+\frac{1}{2}}^{n}:=-\frac{F\left(u_{j}^{n}, u_{j+1}^{n}\right)-F\left(u_{j}^{n}, u_{j}^{n}\right)}{u_{j+1}^{n}-u_{j}^{n}} \quad \text { and } \quad D_{j-\frac{1}{2}}^{n}:=\frac{F\left(u_{j}^{n}, u_{j}^{n}\right)-F\left(u_{j-1}^{n}, u_{j}^{n}\right)}{u_{j}^{n}-u_{j-1}^{n}} .
$$

Next, we rewrite the cell values near the phase boundary. With (3.3), (3.4), and (4.1a) we get

$$
\begin{aligned}
u_{m(n+1)-1}^{n+1}= & u_{m^{-}(n)-1}^{n+1} \\
= & u_{m^{-}(n)-1}^{n}-\frac{\Delta t}{h} D_{m^{-}(n)-\frac{3}{2}}^{n}\left(u_{m^{-}(n)-1}^{n}-u_{m^{-}(n)-2}^{n}\right) \\
& \quad+\Delta m^{-}(n) S_{-}^{n}\left(u_{m(n)-1}^{n}-u_{m(n)-2}^{n}\right),
\end{aligned}
$$




$$
\begin{aligned}
u_{m(n+1)+1}^{n+1}= & u_{m^{+}(n)+1}^{n+1} \\
= & u_{m^{+}(n)+1}^{n}+\frac{\Delta t}{h} C_{m^{+}(n)+\frac{3}{2}}^{n}\left(u_{m^{+}(n)+2}^{n}-u_{m^{+}(n)+1}^{n}\right) \\
& \quad-\frac{\Delta t}{h} D_{m(n)+\frac{1}{2}}^{n}\left(u_{m(n)+1}^{n}-\varphi\left(u_{m(n)-1}^{n}\right)\right) \\
& \quad-\Delta m^{+}(n) S_{+}^{n}\left(u_{m(n)+2}^{n}-u_{m(n)+1}^{n}\right)
\end{aligned}
$$

with

$$
\begin{aligned}
& C_{m^{+}(n)+\frac{3}{2}}^{n}:=-\frac{1}{2+\Delta m^{-}(n)-\alpha^{n+1}} \frac{F\left(u_{m^{+}(n)+1}^{n}, u_{m^{+}(n)+2}^{n}\right)-F\left(u_{m^{+}(n)+1}^{n}, u_{m^{+}(n)+1}^{n}\right)}{u_{m^{+}(n)+2}^{n}-u_{m^{+}(n)+1}^{n}}, \\
& D_{m(n)+\frac{1}{2}}^{n}:= \begin{cases}\frac{\bar{a}\left(u_{m(n)+1}^{n}, \varphi\left(u_{m(n)-1}^{n}\right)\right)-s^{n}}{2+\Delta m^{-}(n)-\alpha^{n+1}} & \text { for } u_{m(n)+1}^{n}<\varphi^{\sharp}\left(u_{m(n)-1}^{n}\right), \\
0 & \text { for } u_{m(n)+1}^{n} \geq \varphi^{\sharp}\left(u_{m(n)-1}^{n}\right),\end{cases} \\
& D_{m^{-}(n)-\frac{3}{2}}^{n}:=\frac{1}{1+\Delta m^{+}(n)+\alpha^{n+1}} \frac{F\left(u_{m^{-}(n)-1}^{n}, u_{m^{-}(n)-1}^{n}\right)-F\left(u_{m^{-}(n)-2}^{n}, u_{m^{-}(n)-1}^{n}\right)}{u_{m^{-}(n)-1}^{n}-u_{m^{-}(n)-2}^{n}}, \\
& S_{-}^{n}:=1-\frac{1}{1+\alpha^{n+1}}\left(1+\frac{\Delta t}{h} \bar{a}\left(u_{m(n)-2}^{n}, u_{m(n)-1}^{n}\right)\right),
\end{aligned}
$$

and

$$
S_{+}^{n}:=1-\frac{1}{2-\alpha^{n+1}}\left(1-\frac{\Delta t}{h} \bar{a}\left(u_{m(n)+1}^{n}, u_{m(n)+2}^{n}\right)\right) .
$$

We use the local Lipschitz-continuity of the numerical flux to bound the generalized Harten coefficients provided we have a uniform $L^{\infty}$-bound on the approximate solution. To ease the notation we set $C_{m(n)-\frac{1}{2}}^{n}=C_{m(n)+\frac{1}{2}}^{n}=D_{m(n)-\frac{1}{2}}^{n}=0$.

LEMMA 5.2. Let the assumptions of Theorem 4.4 and

$$
-M \leq u_{h}(x, t) \leq M, \quad(x, t) \in \mathbb{R} \times[0, T]
$$

be satisfied.

Then we have

$$
\frac{\Delta t}{h} C_{j+\frac{1}{2}}^{n}, \quad \frac{\Delta t}{h} D_{j+\frac{1}{2}}^{n}, \quad \Delta m^{-}(n) S_{-}^{n}, \quad \Delta m^{+}(n) S_{+}^{n} \in\left[0, \frac{1}{2}\right]
$$

for all $h>0, j \in \mathbb{Z}$, and $n \in\{0, \ldots, N\}$.

Proof. By using (3.5), (4.1b) and (4.1c) we get the result for all values except $D_{m(n)+\frac{1}{2}}^{n}$. For the exceptional value $D_{m(n)+\frac{1}{2}}^{n}$ we make a distinction of cases: The case $u_{m(n)+1}^{n} \geq \varphi^{\sharp}\left(u_{m(n)-1}^{n}\right)$ is trivial. In the case $u_{m(n)+1}^{n}<\varphi^{\sharp}\left(u_{m(n)-1}^{n}\right)$ we can deduce from (2.1) the positivity of $D_{m(n)+\frac{1}{2}}^{n}$. We get the inequality $\frac{\Delta t}{h} D_{m(n)+\frac{1}{2}}^{n} \leq \frac{1}{2}$, since $s^{n}$ and $\bar{a}(\cdot, \cdot)$ are bounded by $\hat{C}$ from $(4.1 \mathrm{c})$.

5.3. A Uniform $L^{\infty}$-Bound for the Approximate Solution. Lemma 5.3. Let the assumptions of Theorem 4.4 be fulfilled.

Then the following estimate holds for all $h>0$ and $t \in[0, T]$ :

$$
\left\|u_{h}(\cdot, t)\right\|_{L^{\infty}(\mathbb{R})} \leq M:=\max (1, L)\left\|u_{0}\right\|_{L^{\infty}(\mathbb{R})} .
$$


Proof. In view of $(3.2)$ it is obvious that $\left\|u_{h}^{0}\right\|_{L^{\infty}(\mathbb{R})} \leq\left\|u_{0}\right\|_{L^{\infty}(\mathbb{R})} \leq M$ holds. From (5.3) we get for all $j \in J(n) \cap J(n+1)$

$$
\begin{aligned}
\left|u_{j}^{n+1}\right| & \leq\left(1-\frac{\Delta t}{h} C_{j+\frac{1}{2}}^{n}-\frac{\Delta t}{h} D_{j-\frac{1}{2}}^{n}\right)\left|u_{j}^{n}\right|+\frac{\Delta t}{h} C_{j+\frac{1}{2}}^{n}\left|u_{j+1}^{n}\right|+\frac{\Delta t}{h} D_{j-\frac{1}{2}}^{n}\left|u_{j-1}^{n}\right| \\
& \leq \max \left\{\left|u_{j-1}^{n}\right|,\left|u_{j}^{n}\right|,\left|u_{j+1}^{n}\right|\right\} .
\end{aligned}
$$

With formula (5.4a) and Lemma 5.2 we get also

$$
\begin{aligned}
\left|u_{m(n+1)-1}^{n+1}\right|= & \left|u_{m^{-}(n)-1}^{n+1}\right| \\
\leq & \left(1-\frac{\Delta t}{h} D_{m^{-}(n)-\frac{3}{2}}^{n}-\Delta m^{-}(n) S_{-}^{n}\right)\left|u_{m^{-}(n)-1}^{n}\right| \\
& \quad+\frac{\Delta t}{h} D_{m^{-}(n)-\frac{3}{2}}^{n}\left|u_{m^{-}(n)-2}^{n}\right|+\Delta m^{-}(n) S_{-}^{n}\left|u_{m(n)-1}^{n}\right| \\
\leq & \max \left\{\left|u_{m^{-}(n)-1}^{n}\right|,\left|u_{m^{-}(n)-2}^{n}\right|,\left|u_{m(n)-1}^{n}\right|\right\} .
\end{aligned}
$$

From (5.6) and (5.7) we get by induction

$$
\left|u_{j}^{n}\right| \leq\left\|u_{h}^{0}\right\|_{L^{\infty}\left(-\infty, p^{0}\right)} \leq\left\|u_{0}\right\|_{L^{\infty}\left(-\infty, p^{0}\right)} \quad \text { for all } j \leq m(n)-1 \text { and } n \in\{0, \ldots, N\} .
$$

We proceed with the cell values right to the phase boundary, i.e., $j \geq m(n)+1$. With the last formula and (2.8a) we immediately get

$$
\left|\varphi\left(u_{m(n)-1}^{n}\right)\right| \leq|\varphi(0)|+L\left|u_{m(n)-1}^{n}-0\right| \leq L\left\|u_{0}\right\|_{L^{\infty}\left(-\infty, p^{0}\right)} .
$$

From (5.4b) and in view of Remark 3.3 we get

$$
\begin{aligned}
& \left|u_{m(n+1)+1}^{n+1}\right|=\left|u_{m^{+}(n)+1}^{n+1}\right| \\
& \leq\left(1-\frac{\Delta t}{h} C_{m^{+}(n)+\frac{3}{2}}^{n}-\left(1-\Delta m^{+}(n)\right) \frac{\Delta t}{h} D_{m(n)+\frac{1}{2}}^{n}-\Delta m^{+}(n) S_{+}^{n}\right)\left|u_{m^{+}(n)+1}^{n}\right| \\
& +\Delta m^{+}(n)\left(S_{+}^{n}-\frac{\Delta t}{h} D_{m(n)+\frac{1}{2}}^{n}\right)\left|u_{m(n)+1}^{n}\right| \\
& +\frac{\Delta t}{h} C_{m^{+}(n)+\frac{3}{2}}^{n}\left|u_{m^{+}(n)+2}^{n}\right|+\frac{\Delta t}{h} D_{m(n)+\frac{1}{2}}^{n}\left|\varphi\left(u_{m(n)-1}^{n}\right)\right| \\
& \leq \max \left\{\left|u_{m^{+}(n)+1}^{n}\right|,\left|u_{m^{+}(n)+2}^{n}\right|,\left|u_{m(n)+1}^{n}\right|,\left|\varphi\left(u_{m(n)-1}^{n}\right)\right|\right\} \text {. }
\end{aligned}
$$

From this and again (5.6), we obtain by induction

$$
\left|u_{j}^{n}\right| \leq \max (1, L)\left\|u_{0}\right\|_{L^{\infty}} \quad \text { for all } j \geq m(n)+1 \text { and } n \in\{0, \ldots, N\}
$$

Combining the results (5.8) and (5.9) implies (5.5). 
5.4. A Uniform Total Variation Bound for the Approximate Solution. We derive a uniform estimate on the total variation (TV) of $u_{h}$. Similarly as in Section 5.3 it is possible to estimate the total variation left to the phase boundary only using values left to it.

LEMMA 5.4. Let the assumptions of Theorem 4.4 be fulfilled.

Then the following formula holds for all $\mathcal{N} \in\{1, \ldots, N\}$

$$
\sum_{j<m(\mathcal{N})-1}\left|u_{j+1}^{\mathcal{N}}-u_{j}^{\mathcal{N}}\right|+\sum_{n=0}^{\mathcal{N}-1}\left|u_{m(n+1)-1}^{n+1}-u_{m(n)-1}^{n}\right| \leq \sum_{j<m(0)-1}\left|u_{j+1}^{0}-u_{j}^{0}\right| .
$$

Proof. Let us first define $w_{j+\frac{1}{2}}^{n}=\left|u_{j+1}^{n}-u_{j}^{n}\right|$. We get

$$
w_{j+\frac{1}{2}}^{n+1} \leq\left(1-\frac{\Delta t}{h} D_{j+\frac{1}{2}}^{n}-\frac{\Delta t}{h} C_{j+\frac{1}{2}}^{n}\right) w_{j+\frac{1}{2}}^{n}+\frac{\Delta t}{h} D_{j-\frac{1}{2}}^{n} w_{j-\frac{1}{2}}^{n}+\frac{\Delta t}{h} C_{j+\frac{3}{2}}^{n} w_{j+\frac{3}{2}}^{n} .
$$

Then we sum over $j<m(n)-1$ and get

$$
\begin{aligned}
& \sum_{j<m(n+1)-1} w_{j+\frac{1}{2}}^{n+1}=\sum_{j<m^{-}(n)-1} w_{j+\frac{1}{2}}^{n+1} \\
& \quad \leq \sum_{j<m^{-}(n)-1} w_{j+\frac{1}{2}}^{n}-\frac{\Delta t}{h} D_{m^{-}(n)-\frac{3}{2}}^{n} w_{m^{-}(n)-\frac{3}{2}}^{n}-\frac{\Delta t}{h} \underbrace{C_{m^{-}(n)-\frac{1}{2}}^{n}}_{=0} w_{m^{-}(n)-\frac{1}{2}}^{n} \\
& \quad=\sum_{j<m(n)-1} w_{j+\frac{1}{2}}^{n}-\frac{\Delta t}{h} D_{m^{-}(n)-\frac{3}{2}}^{n} w_{m^{-}(n)-\frac{3}{2}}^{n}-\Delta m^{-}(n) w_{m(n)-\frac{3}{2}}^{n} \\
& \leq \sum_{j<m(n)-1} w_{j+\frac{1}{2}}^{n}-\left|u_{m(n+1)-1}^{n+1}-u_{m(n)-1}^{n}\right| .
\end{aligned}
$$

The last inequality follows directly from (5.4a), since

$$
\begin{aligned}
\left|u_{m(n+1)-1}^{n+1}-u_{m(n)-1}^{n}\right| \leq & \frac{\Delta t}{h} D_{m^{-}(n)-\frac{3}{2}}^{n}\left|u_{m^{-}(n)-1}^{n}-u_{m^{-}(n)-2}^{n}\right| \\
& \quad+\Delta m^{-}(n) S_{-}^{n}\left|u_{m(n)-1}^{n}-u_{m(n)-2}^{n}\right| \\
\leq & \frac{\Delta t}{h} D_{m^{-}(n)-\frac{3}{2}}^{n} w_{m^{-}(n)-\frac{3}{2}}^{n}+\Delta m^{-}(n) w_{m(n)-\frac{3}{2}}^{n} .
\end{aligned}
$$

Finally, we sum over $0 \leq n \leq \mathcal{N}-1$ and get the result by rearranging the terms

$$
\sum_{j<m(\mathcal{N})-1} w_{j+\frac{1}{2}}^{\mathcal{N}}+\sum_{n=0}^{\mathcal{N}-1}\left|u_{m(n+1)-1}^{n+1}-u_{m(n)-1}^{n}\right| \leq \sum_{j<m(0)-1} w_{j+\frac{1}{2}}^{0} .
$$

口

Let us now recall the definition of the total variation. For $v \in L^{1}(\mathbb{R})$ we define

$$
\operatorname{TV}(v):=\lim _{h \rightarrow 0} \int_{\mathbb{R}} \frac{1}{h}|v(x+h)-v(x)| \mathrm{d} x .
$$

A function $v$ is total variation bounded if $\operatorname{TV}(v)<\infty$. For a piecewise constant function as $u_{h}^{n}$, we get

$$
\operatorname{TV}\left(u_{h}^{n}\right)=\sum_{j \notin\{m(n)-1, m(n)\}}\left|u_{j+1}^{n}-u_{j}^{n}\right|+\left|u_{m(n)+1}^{n}-u_{m(n)-1}^{n}\right| .
$$


We further introduce the operator $\mathrm{TV}^{*}$ as

$\mathrm{TV}^{*}\left(u_{h}^{n}\right):=\sum_{j \notin\{m(n)-1, m(n)\}}\left|u_{j+1}^{n}-u_{j}^{n}\right|+\left|u_{m(n)+1}^{n}-\varphi\left(u_{m(n)-1}^{n}\right)\right|+\left|\varphi\left(u_{m(n)-1}^{n}\right)-u_{m(n)-1}^{n}\right|$.

We have the trivial relation

$$
\operatorname{TV}\left(u_{h}^{n}\right) \leq \operatorname{TV}^{*}\left(u_{h}^{n}\right)
$$

However, the advantage of $\mathrm{TV}^{*}$ is that the difference $\mathrm{TV}^{*}\left(u_{h}^{n+1}\right)-\mathrm{TV}^{*}\left(u_{h}^{n}\right)$ can be estimated independently of $u_{m(n)+1}^{n}$, see proof of Lemma 5.5 below. Note that the maximally possible increase in total variation is not realized within one time step but only in the asymptotic regime. That makes it hard to control $\operatorname{TV}\left(u_{h}^{n}\right)$ directly.

LEMMA 5.5. Let the assumptions from Theorem 4.4 be fulfilled.

Then we have for all $h>0$ and $n \in\{0, \ldots, N\}$

$$
\operatorname{TV}\left(u_{h}^{n}\right) \leq(1+2 L) \mathrm{TV}^{*}\left(u_{0}\right)
$$

Here, $\mathrm{TV}^{*}\left(u_{0}\right)$ is given by

$$
\operatorname{TV}^{*}\left(u_{0}\right):=\operatorname{TV}\left(u_{0}\right)-\left|u_{+}-u_{-}\right|+\left|u_{+}-\varphi\left(u_{-}\right)\right|+\left|\varphi\left(u_{-}\right)-u_{-}\right|, \quad u_{ \pm}=u_{0}(0 \pm) .
$$

Proof. The following proof exploits again the fact that the scheme can be written in Harten form. For $n \geq 0$ we have

$$
\begin{aligned}
& \operatorname{TV}^{*}\left(u_{h}^{n+1}\right)-\operatorname{TV}^{*}\left(u_{h}^{n}\right) \\
= & \sum_{j \in \mathbb{Z} \backslash\left[m^{-}(n)-1, m^{+}(n)\right]}\left(\left|u_{j+1}^{n+1}-u_{j}^{n+1}\right|-\left|u_{j+1}^{n}-u_{j}^{n}\right|\right) \\
& +\left|u_{m(n+1)+1}^{n+1}-\varphi\left(u_{m(n+1)-1}^{n+1}\right)\right|-\left|u_{m(n)+1}^{n}-\varphi\left(u_{m(n)-1}^{n}\right)\right|-\Delta m^{+}(n)\left|u_{m(n)+2}^{n}-u_{m(n)+1}^{n}\right| \\
& +\left|\varphi\left(u_{m(n+1)-1}^{n+1}\right)-u_{m(n+1)-1}^{n+1}\right|-\left|\varphi\left(u_{m(n)-1}^{n}\right)-u_{m(n)-1}^{n}\right|-\Delta m^{-}(n)\left|u_{m(n)-1}^{n}-u_{m(n)-2}^{n}\right| \\
& +\Delta m^{+}(n)\left|u_{m(n+1)-1}^{n+1}-u_{m(n+1)-2}^{n+1}\right|+\Delta m^{-}(n)\left|u_{m(n+1)+2}^{n+1}-u_{m(n+1)+1}^{n+1}\right| \\
= & : L_{1}+L_{2}+L_{3}+L_{4} .
\end{aligned}
$$

For $L_{1}$ we get from $(5.3)$

$$
\begin{aligned}
L_{1} \leq & -\frac{\Delta t}{h} C_{m^{+}(n)+\frac{3}{2}}^{n}\left|u_{m^{+}(n)+2}^{n}-u_{m^{+}(n)+1}^{n}\right|+\frac{\Delta t}{h} D_{m(n)+\frac{1}{2}}^{n}\left|u_{m(n)+1}^{n}-\varphi\left(u_{m(n)-1}^{n}\right)\right| \\
& +\Delta m^{+}(n) S_{+}^{n}\left|u_{m(n)+2}^{n}-u_{m(n)+1}^{n}\right| \\
& -\frac{\Delta t}{h} D_{m^{-}(n)-\frac{3}{2}}^{n}\left|u_{m^{-}(n)-1}^{n}-u_{m^{-}(n)-2}^{n}\right|+\Delta m^{-}(n) S_{-}^{n}\left|u_{m(n)-1}^{n}-u_{m(n)-2}^{n}\right| \\
= & : R_{1}+R_{2}+R_{3} .
\end{aligned}
$$

For the terms $L_{2}$ and $L_{3}$ we use the following representation of $\varphi\left(u_{m(n+1)-1}^{n+1}\right)$

$$
\varphi\left(u_{m(n+1)-1}^{n+1}\right)=\varphi\left(u_{m(n)-1}^{n}\right)-T_{\varphi}^{n}\left(u_{m(n+1)-1}^{n+1}-u_{m(n)-1}^{n}\right)
$$


with

$$
T_{\varphi}^{n}:=\frac{\varphi\left(u_{m(n+1)-1}^{n+1}\right)-\varphi\left(u_{m(n)-1}^{n}\right)}{u_{m(n+1)-1}^{n+1}-u_{m(n)-1}^{n}} .
$$

From (2.8b) it is clear that $T_{\varphi}^{n} \in[-L, 0]$. To further examine $L_{2}$, we use (5.4b), (5.12), and get

$$
L_{2} \leq-R_{1}-R_{2}+\left|T_{\varphi}^{n}\right|\left|u_{m(n+1)-1}^{n+1}-u_{m(n)-1}^{n}\right| .
$$

With (5.4a) and (5.12) we get

$$
L_{3} \leq-R_{3}+\left|T_{\varphi}^{n}\right|\left|u_{m(n+1)-1}^{n+1}-u_{m(n)-1}^{n}\right| .
$$

By the definition of the scheme, see (5.4a) and (5.4b), we have $L_{4}=0$. Finally, we sum all terms from (5.11), (5.13), (5.14) and use Lemma 5.2 to get

$$
\mathrm{TV}^{*}\left(u_{h}^{n+1}\right)-\mathrm{TV}^{*}\left(u_{h}^{n}\right) \leq 2 L\left|u_{m(n+1)-1}^{n+1}-u_{m(n)-1}^{n}\right| .
$$

By summing all time steps up to $N \geq 0$ we get the final result:

$$
\begin{aligned}
\mathrm{TV}^{*}\left(u_{h}^{N+1}\right) & =\mathrm{TV}^{*}\left(u_{h}^{0}\right)+\sum_{n=0}^{N}\left(\mathrm{TV}^{*}\left(u_{h}^{n+1}\right)-\mathrm{TV}^{*}\left(u_{h}^{n}\right)\right) \\
& \leq \mathrm{TV}^{*}\left(u_{h}^{0}\right)+2 L \sum_{n=0}^{N}\left|u_{m(n+1)-1}^{n+1}-u_{m(n)-1}^{n}\right| \\
& \leq(1+2 L) \mathrm{TV}^{*}\left(u_{h}^{0}\right) .
\end{aligned}
$$

For the last inequality we used Lemma 5.4. Finally, we get (5.10).

5.5. Continuity in Time. Lemma 5.6 (Continuity in Time). Under the assumptions of Theorem 4.4, there is a $C>0$ such that for all $0 \leq t_{1}<t_{2} \leq T$

$$
\left\|u_{h}\left(\cdot, t_{2}\right)-u_{h}\left(\cdot, t_{1}\right)\right\|_{L^{1}(\mathbb{R})} \leq C\left(t_{2}-t_{1}\right) .
$$

Proof. Thanks to the Harten form of the scheme the proof follows exactly the same lines as for monotone schemes, see e.g. [17], Theorem 2.3.9.

From Lemmas 5.3, 5.5, and 5.6 and Helly's theorem imply that there is a subsequence of $\left\{u_{h}\right\}_{h>0}$ and a function $u \in V$ such that the first assertion of Theorem 4.4 holds. In the following we will show that the limit function $u$ is a $(\bar{\eta}, \varphi)$-solution of (1.1).

5.6. A Cell Entropy Inequality. In this section we will prove cell entropy inequalities, which will be used in Section 5.7 to prove that $u_{h}$ converges to a function that obeys (2.7).

Lemma 5.7 (Cell Entropy Inequality). Under the assumptions of Theorem 4.4, the entropy inequality

$$
\bar{\eta}\left(u_{j}^{n+1}\right) \leq \bar{\eta}\left(u_{j}^{n}\right)-\frac{\Delta t}{h}\left(q_{j+\frac{1}{2}}^{n}-q_{j-\frac{1}{2}}^{n}\right)
$$


holds for all $j \in J(n) \cap J(n+1), n \in\{0, \ldots, N\}$, and $h>0$. Furthermore, we have

$$
\begin{aligned}
& \left(1+\Delta m^{+}(n)+\alpha^{n+1}\right) \bar{\eta}\left(u_{m(n)-1}^{n+1}\right) \\
& \quad \leq\left(1+\alpha^{n}\right) \bar{\eta}\left(u_{m(n)-1}^{n}\right)+\Delta m^{-}(n) \bar{\eta}\left(u_{m(n)-2}^{n}\right)-\frac{\Delta t}{h}\left(q_{m(n)}^{n}-q_{m^{-}(n)-\frac{3}{2}}^{n}\right)
\end{aligned}
$$

and

$$
\begin{aligned}
& \left(2+\Delta m^{-}(n)-\alpha^{n+1}\right) \bar{\eta}\left(u_{m(n)+1}^{n+1}\right) \\
& \leq\left(2-\alpha^{n}\right) \bar{\eta}\left(u_{m(n)+1}^{n}\right)+\Delta m^{+}(n) \bar{\eta}\left(u_{m(n)+2}^{n}\right)-\frac{\Delta t}{h}\left(q_{m^{+}(n)+\frac{3}{2}}^{n}-q_{m(n)}^{n}\right) .
\end{aligned}
$$

Here, the entropy fluxes are defined by

$$
q_{j+\frac{1}{2}}^{n}=Q\left(u_{j}^{n}, u_{j+1}^{n}\right) \quad \text { and } \quad q_{m(n)}^{n}=\bar{q}\left(u_{m(n)-1}^{n}\right)-s^{n} \bar{\eta}\left(u_{m(n)-1}^{n}\right) .
$$

Proof. The proof for (5.15) can be done as for monotone schemes, see e.g. [17], Proposition 3.3.26.

We show only (5.17) for $\Delta m(n)=1$, which is the most complicated case. The case $\Delta m(n) \neq 1$ follows the same lines. Using (3.11b) we get

$$
u_{m(n+1)+1}^{n+1}=\frac{T_{1}+T_{2}+\left(1-\alpha^{n+1}\right)\left(T_{3}+T_{4}\right)}{2\left(2-\alpha^{n+1}\right)}
$$

with

$$
\begin{aligned}
& T_{1}:=u_{m(n)+2}^{n}-2 \frac{\Delta t}{h}\left(f_{m(n)+\frac{5}{2}}^{n}-f\left(u_{m(n)+2}^{n}\right)\right), \\
& T_{2}:=u_{m(n)+2}^{n}-2 \frac{\Delta t}{h}\left(f\left(u_{m(n)+2}^{n}\right)-f_{m(n)+\frac{3}{2}}^{n}\right), \\
& T_{3}:=u_{m(n)+1}^{n}-\frac{2}{1-\alpha^{n+1}} \frac{\Delta t}{h}\left(f\left(u_{m(n)+1}^{n}\right)-s^{n} u_{m(n)+1}^{n}-h^{n}\right), \\
& T_{4}:=u_{m(n)+1}^{n}-\frac{2}{1-\alpha^{n+1}} \frac{\Delta t}{h}\left(f_{m(n)+\frac{3}{2}}^{n}-f\left(u_{m(n)+1}^{n}\right)\right) .
\end{aligned}
$$

As the next step, we state the inequalities

$$
\begin{aligned}
& \eta\left(T_{1}\right) \leq \eta\left(u_{m(n)+2}^{n}\right)-2 \frac{\Delta t}{h}\left(q_{m(n)+\frac{5}{2}}^{n}-q\left(u_{m(n)+2}^{n}\right)\right) \\
& \eta\left(T_{2}\right) \leq \eta\left(u_{m(n)+2}^{n}\right)-2 \frac{\Delta t}{h}\left(q\left(u_{m(n)+2}^{n}\right)-q_{m(n)+\frac{3}{2}}^{n}\right) \\
& \eta\left(T_{3}\right) \leq \eta\left(u_{m(n)+1}^{n}\right)-\frac{2}{1-\alpha^{n+1}} \frac{\Delta t}{h}\left(q\left(u_{m(n)+1}^{n}\right)-s^{n} \eta\left(u_{m(n)+1}^{n}\right)-q_{m(n)}^{n}\right) \\
& \eta\left(T_{4}\right) \leq \eta\left(u_{m(n)+1}^{n}\right)-\frac{2}{1-\alpha^{n+1}} \frac{\Delta t}{h}\left(q_{m(n)+\frac{3}{2}}^{n}-q\left(u_{m(n)+1}^{n}\right)\right) .
\end{aligned}
$$

We give the proof of (5.19c), all others inequalities are similar as in the standard situation [17]. In the Laxian case, where $s^{n}=\bar{a}\left(u_{m(n)+1}^{n}, u_{m(n)-1}^{n}\right)$, the inequality is trivially fulfilled, since $T_{3}=u_{m(n)+1}^{n}$ and the wave connecting $u_{m(n)-1}^{n}$ and $u_{m(n)+1}^{n}$ fulfils the entropy inequality

$$
s^{n}\left(\eta\left(u_{m(n)-1}^{n}\right)-\eta\left(u_{m(n)+1}^{n}\right)\right)-\left(q\left(u_{m(n)-1}^{n}\right)-q\left(u_{m(n)+1}^{n}\right)\right) \leq 0 .
$$


In the undercompressive case, we set $a=u_{m(n)+1}^{n}, \lambda=\frac{2}{1-\alpha^{n+1}} \frac{\Delta t}{h}$, and $g(b):=\eta\left(a-\lambda\left(f(a)-f(b)-s^{n}(a-b)\right)\right)-\eta(a)+\lambda\left(q(a)-q(b)-s^{n}(\eta(a)-\eta(b))\right)$.

We have to show the inequality $g\left(\varphi\left(u_{m(n)-1}^{n}\right)\right) \leq 0$, which together with (2.10) implies (5.19c). We will prove $g(b) \leq 0$ for the whole interval $b \in\left(-M, \varphi^{\sharp}\left(u_{m(n)-1}^{n}\right)\right)$. Here $M$ is defined as before by $M:=\max (1, L)\left\|u_{0}\right\|_{L^{\infty}}$.

By differentiating $g$ we get

$$
\begin{aligned}
g^{\prime}(b) & =\eta^{\prime}\left(a-\lambda\left(f(a)-f(b)-s^{n}(a-b)\right)\right) \lambda\left(f^{\prime}(b)-s^{n}\right)+\lambda\left(q^{\prime}(b)-s^{n} \eta^{\prime}(b)\right) \\
& =\lambda\left(f^{\prime}(b)-s^{n}\right)\left(\eta^{\prime}\left(a-\lambda\left(f(a)-f(b)-s^{n}(a-b)\right)\right)-\eta^{\prime}(b)\right) \\
& =\lambda\left(f^{\prime}(b)-s^{n}\right) \eta^{\prime \prime}\left(\xi_{1}\right)\left(a-b-\lambda\left(f(a)-f(b)-s^{n}(a-b)\right)\right) \\
& =\lambda\left(f^{\prime}(b)-s^{n}\right) \eta^{\prime \prime}\left(\xi_{1}\right)\left(1-\lambda\left(f^{\prime}\left(\xi_{2}\right)-s^{n}\right)\right)(a-b),
\end{aligned}
$$

where

$$
\xi_{1} \in I\left(a-\lambda\left(f(a)-f(b)-s^{n}(a-b)\right), b\right) \quad \text { and } \quad \xi_{2} \in I(a, b)
$$

are values arising in the mean value theorem. Here, $I(a, b)=[a, b] \cup[b, a]$. By Lemma 5.1, (3.5), and

$$
\left|\lambda\left(f^{\prime}\left(\xi_{2}\right)-s^{n}\right)\right| \leq \frac{2}{8 \hat{C}\left(1-\alpha^{n+1}\right)}\left(\left|f^{\prime}\left(\xi_{2}\right)\right|+\left|s^{n}\right|\right) \leq \frac{2 \cdot 2 \hat{C}}{8 \hat{C}^{\frac{3}{4}}}<1
$$

we get $g^{\prime}(b)(a-b) \geq 0$ for $b \in\left(-M, \varphi^{\sharp}\left(u_{m(n)-1}^{n}\right)\right)$. As we have $g(a)=0$, we get $g(b) \leq 0$ for $b \in\left(-M, \varphi^{\sharp}\left(u_{m(n)-1}^{n}\right)\right)$.

Putting (5.19a)-(5.19d) together, we get

$$
\begin{gathered}
\left(2-\alpha^{n+1}\right) \eta\left(u_{m(n)+1}^{n+1}\right) \leq \frac{1}{2}\left(\eta\left(T_{1}\right)+\eta\left(T_{2}\right)+\left(1-\alpha^{n+1}\right)\left(\eta\left(T_{3}\right)+\eta\left(T_{4}\right)\right)\right) \\
=\left(2-\alpha^{n}\right) \eta\left(u_{m(n)+1}^{n}\right)+\eta\left(u_{m(n)+2}^{n}\right)-\frac{\Delta t}{h}\left(q_{m(n)+\frac{5}{2}}^{n}-q_{m(n)}^{n}\right) .
\end{gathered}
$$

The proof for (5.16) is done in the same way.

5.7. Lax-Wendroff Theorem. In this section we state a Lax-Wendroff theorem for the scheme.

THEOREM 5.8 (Lax-Wendroff). Let the assumptions of Theorem 4.4 be fulfilled. If the sequence $\left\{u_{h}\right\}_{h>0}$ converges to a limit function $u \in V$, then the limit function $u$ is a weak solution in the sense of (2.6) and an entropy solution in the sense of (2.7).

Proof. For the proof we refer to [10], which states a result for general quasiuniform grid. We point out that the computational grid introduced in Section 3 fulfils the requirements of a quasi-uniform grid. 
5.8. Recovery of the Phase Boundary Position. To prove Theorem 4.4 completely it remains to verify the pointwise properties 2 . and 3 . in Definition 2.3. In this section we construct the function $p:[0, T] \rightarrow \mathbb{R}$ from the Definition 2.3. We will verify (2.13) in the next section.

We define a continuous version $p_{h}:[0, T] \rightarrow \mathbb{R}$ of the position of the discrete phase boundary $\left(p^{n}\right)_{n \geq 0}$ from (3.1) by

$$
p_{h}(t)=p^{n}+s^{n}\left(t-t^{n}\right) \quad \text { for all } t \in\left[t^{n}, t^{n+1}\right) .
$$

The set $\left\{p_{h}:[0, T] \rightarrow \mathbb{R}\right\}_{h>0}$ is uniformly Lipschitz-continuous, since $s^{n}$ defined in (3.3) is bounded, since Lemma 5.3 states that $\left\{u_{m(n)-1}^{n}\right\}_{n}$ is bounded. By the Arzela-Ascoli theorem we can select a subsequence with

$$
p_{h} \longrightarrow p \quad \text { in } C^{0}([0, T]) \text { for } h \rightarrow 0 .
$$

5.9. Trace Values. After the construction of $p$ the trace values $u_{-}$and $u_{+}$at the phase boundary are well-defined. The next definition introduces more quantities defined on the phase boundary.

Definition 5.9. Let $u \in V$ be the limit of $\left\{u_{h}\right\}_{h>0}$ and $p \in \operatorname{Lip}([0, T])$ the limit of $\left\{p_{h}\right\}_{h>0}$.

We define the phase boundary speed $s \in L^{1}([0, T])$, the phase boundary flux $H \in$ $L^{1}([0, T])$ and the entropy dissipation $D \in L^{1}([0, T])$ by the following formulas:

$$
\begin{aligned}
s(t) & =\bar{a}\left(u_{+}(t), u_{-}(t)\right), \\
H(t) & =f\left(u_{-}(t)\right)-s(t) u_{-}(t), \\
D(t) & =-s(t)\left(\bar{\eta}\left(u_{+}(t)\right)-\bar{\eta}\left(u_{-}(t)\right)\right)+\left(\bar{q}\left(u_{+}(t)\right)-\bar{q}\left(u_{-}(t)\right)\right) .
\end{aligned}
$$

All quantities defined in Definition 5.9 have discrete analogons, which we define in the next definition.

Definition 5.10. We define the discrete traces values $u_{h,-}^{*}, u_{h,+}^{*} \in L^{1}([0, T])$, the discrete phase boundary speed $s_{h}^{*} \in L^{1}([0, T])$, the discrete phase boundary flux $H_{h}^{*} \in L^{1}([0, T])$ and the discrete phase boundary dissipation $D_{h}^{*} \in L^{1}([0, T])$ by

$$
\begin{aligned}
u_{h,-}^{*}(t) & =u_{m(n)-1}^{n}, \\
u_{h,+}^{*}(t) & =u_{m(n)+1}^{n}, \\
s_{h}^{*}(t) & =s^{n}, \\
H_{h}^{*}(t) & =h^{n}=f\left(u_{m(n)-1}^{n}\right)-s^{n} u_{m(n)-1}^{n}, \\
D_{h}^{*}(t)=\frac{h}{\Delta t}\left(\alpha^{n+1} \eta\left(u_{m(n)-1}^{n+1}\right)+\left(1-\alpha^{n+1}\right) \eta\left(u_{m(n)+1}^{n+1}\right)-\alpha^{n} \eta\left(u_{m(n)-1}^{n}\right)\right. & \left.\quad-\left(1-\alpha^{n}\right) \eta\left(u_{m(n)+1}^{n}\right)\right)+q\left(u_{m(n)+1}^{n}\right)-q\left(u_{m(n)-1}^{n}\right)
\end{aligned}
$$

for $t \in\left[t^{n}, t^{n+1}\right)$. The next lemma provides convergence properties of these quantities:

Lemma 5.11. Under the assumptions of Theorem 4.4, there are functions $u_{-}^{*}$, $u_{+}^{*}, H^{*}, s^{*}, D^{*} \in L^{1}([0, T])$, such that for a subsequence it is true that

$$
u_{h,-}^{*} \rightarrow u_{-}^{*}, \quad u_{h,+}^{*} \rightarrow u_{+}^{*}, \quad s_{h}^{*} \rightarrow s^{*}, \quad H_{h}^{*} \rightarrow H^{*}, \quad \text { and } \quad D_{h}^{*} \rightarrow D^{*}
$$


for $h \rightarrow 0$ in $L^{1}([0, T])$. Furthermore, we have for almost all $t \in[0, T]$

$$
D^{*}(t)=-\bar{a}\left(u_{-}^{*}(t), u_{+}^{*}(t)\right)\left(\bar{\eta}\left(u_{+}^{*}(t)\right)-\bar{\eta}\left(u_{-}^{*}(t)\right)\right)+\left(\bar{q}\left(u_{+}^{*}(t)\right)-\bar{q}\left(u_{-}^{*}(t)\right)\right) .
$$

Proof. Note that $u_{h,-}^{*}, u_{h,+}^{*}, s_{h}^{*}, H_{h}^{*}$, and $D_{h}^{*}$ are TV-bound by using Lemma 5.4 . Helly's theorem selects a converging subsequence. For the convergence of $D_{h}^{*}$ and the limit representation (5.20) we need (3.5) to control the term $\frac{h}{\Delta t}$ and the iteration formula (3.6) for the representation.

In the next step we analyze the relationship between the $*$-quantities in Lemma 5.11 and corresponding trace quantities of the limit function $u$ in Definition 5.9.

LEMMA 5.12. Under the assumptions of Theorem 4.4, we have

$$
s(t)=s^{*}(t), \quad H(t)=H^{*}(t), \quad \text { and } \quad D(t) \leq D^{*}(t)
$$

for almost all $t \in[0, T]$.

Proof.

$\boldsymbol{s}(\boldsymbol{t})=\boldsymbol{s}^{*}(\boldsymbol{t}):$ From the facts that $s_{h}^{*}=\frac{\partial p_{h}}{\partial t} \rightarrow s^{*}$ in $L^{1}([0, T]), p_{h} \rightarrow p$ in $C^{0}([0, T])$, the existence of $s=\frac{\partial p}{\partial t}$ and the uniform bound of $\left\|s_{h}^{*}\right\|_{L^{\infty}}$, we immediately get the result.

$\boldsymbol{H}(\boldsymbol{t})=\boldsymbol{H}^{*}(\boldsymbol{t})$ : We get for almost all $0 \leq t_{1}<t_{2} \leq T$ the mass balance

$$
\int_{-\infty}^{p_{h}\left(t_{2}\right)} u_{h}\left(x, t_{2}\right) \mathrm{d} x-\int_{-\infty}^{p_{h}\left(t_{1}\right)} u_{h}\left(x, t_{1}\right) \mathrm{d} x=\int_{t^{1}}^{t^{2}} H_{h}^{*}(t) \mathrm{d} t+O(\Delta t) .
$$

In the limit $h \rightarrow 0$ we get by $p_{h} \rightarrow p$ in $C^{0}([0, T])$ and $u_{h}(\cdot, t) \rightarrow u(\cdot, t)$

$$
\int_{-\infty}^{p\left(t_{2}\right)} u\left(x, t_{2}\right) \mathrm{d} x-\int_{-\infty}^{p\left(t_{1}\right)} u\left(x, t_{1}\right) \mathrm{d} x=\int_{t^{1}}^{t^{2}} H^{*}(t) \mathrm{d} t
$$

Since $u$ is a weak solution, we get (5.21) also for $H$ instead of $H^{*}$, what finishes the proof.

$\boldsymbol{D}(\boldsymbol{t}) \leq \boldsymbol{D}^{*}(\boldsymbol{t}):$ To prove this, we introduce a dissipation measure $\hat{D} \in \mathcal{M}(\mathbb{R} \times[0, T])$ by

$$
\hat{D}=\partial_{t} \bar{\eta}(u)+\partial_{x} \bar{q}(u)
$$

We see that $\left.\hat{D}\right|_{x=p(t)}=D \mu_{\{x=p(t)\}}^{1}$. Here $\mu^{1}$ is the one-dimensional Hausdorff measure and $\lambda$ the Lebesgue measure. We have to be more careful in the discrete setting to obtain a similar result. We define

$D_{j}^{n}:=\frac{1}{\Delta t}\left(\bar{\eta}\left(u_{j+1}^{n}\right)-\bar{\eta}\left(u_{j}^{n}\right)\right)+\frac{1}{h}\left(q_{j+\frac{1}{2}}^{n}-q_{j-\frac{1}{2}}^{n}\right)$,

$\hat{D}_{h}^{*}:=\sum_{0 \leq n<N} \sum_{j \notin\{m(n)-1, m(n), m(n)+1\}} D_{j}^{n} \lambda_{\left[x_{j-\frac{1}{2}}, x_{j+\frac{1}{2}}\right) \times\left[t^{n}, t^{n+1}\right)}+D_{h}^{*} \mu_{\left\{x=p_{h}(t)\right\}}^{1}$.

Again we have $\left.\hat{D}_{h}^{*}\right|_{x=p_{h}(t)}=D_{h}^{*} \mu_{\left\{x=p_{h}(t)\right\}}^{1}$. From $\hat{D}_{h}^{*} \leq 0$ (see Lemma 5.7) and $\hat{D}_{h}^{*} \rightarrow \hat{D}$ in the sense of measures the result follows directly. 
口

Let us mention that Lemma 5.12 shows that $p$ fulfils (2.13), which is equivalent to $s(t)=s^{*}(t)$. With the help of the just introduced definitions and lemmas, we can state the main result of this section.

We note that the relation $s(t)=s^{*}(t)$ from Lemma 5.12 is equivalent to (2.13) in the definition of the $(\bar{\eta}, \varphi)$-solutions. It remains to verify condition (2.14).

THEOREM 5.13. Under the assumptions of Theorem 4.4, we have for almost every $t \in[0, T]$ that either

$$
u_{+}(t)=\varphi\left(u_{-}(t)\right) \quad \text { or } \quad u_{+}(t) \geq \varphi^{\sharp}\left(u_{-}(t)\right)
$$

is fulfilled.

Proof. Fix $t \in[0, T]$ and select a subsequence of $\left\{u_{h}\right\}_{h>0}$ such that $u_{h,-}^{*}(t)$, $u_{h,+}^{*}(t), s_{h}^{*}(t)$, and $D_{h}^{*}(t)$ converge. This is possible for almost every $t \in[0, T]$ and we will show (5.22) under these assumptions. Define a sequence $\left\{n_{h}\right\}_{h>0}$ such that

$$
n_{h}=\max \left\{n \in \mathbb{N} \mid t_{h}^{n} \leq t\right\}, \quad t_{h}^{n}=n \Delta t_{h}=\frac{n h}{8 \hat{C}} .
$$

For this sequence we have

$$
u_{h,-}^{*}(t)=u_{m\left(n_{h}\right)-1}^{n_{h}} \rightarrow u_{-}^{*}(t), \quad \ldots \quad \text { for } h \rightarrow 0 .
$$

In the following we will drop the index $h$ for better reading.

Case 1: There are infinitely many $h>0$ such that $u_{m(n)+1}^{n}<\varphi^{\sharp}\left(u_{m(n)-1}^{n}\right)$. In this case we can restrict ourselves to a subsequence of $\left\{u_{h}\right\}_{h>0}$ which fulfils this condition in every step. Recall $H^{*}(t)$ from Definition 5.10 and consider for $t \in[0, T]$ fixed the equation

$$
H_{h}^{*}(t)=f(w)-s_{h}^{*}(t) w
$$

It allows three solutions, namely $w=u_{m(n)-1}^{n}, w=\varphi^{\sharp}\left(u_{m(n)-1}^{n}\right)$ and $w=$ $\varphi\left(u_{m(n)-1}^{n}\right)$. From the continuity of the solutions of (5.23) with respect to the parameters $H_{h}^{*}(t)$ and $s_{h}^{*}(t)$ we get, that the limit equation

$$
H^{*}(t)=f(w)-s^{*}(t) w
$$

allows solutions $w=v, w=\varphi^{\sharp}(v)$, and $w=\varphi(v)$ for some $v \in \mathbb{R}$. From Lemma 5.12 and the fact that $u$ is a weak solution, we know that

$$
u_{-}(t), u_{+}(t) \in\left\{v, \varphi^{\sharp}(v), \varphi(v)\right\} .
$$

Two situations are possible:

$$
\begin{aligned}
& u_{-}(t)=v, \quad u_{+}(t)=\varphi(v) \quad \Longrightarrow \quad \text { first equation of (5.22) is fulfilled. } \\
& u_{-}(t)=v, \quad u_{+}(t)=\varphi^{\sharp}(v) \quad \Longrightarrow \quad \text { second inequality (5.22) is fulfilled. }
\end{aligned}
$$

Case 2: If we do not have infinitely many $h>0$ with $u_{m(n)+1}^{n}<\varphi^{\sharp}\left(u_{m(n)-1}^{n}\right)$ as in Case 1 , then we can choose a subsequence such that $u_{m(n)+1}^{n} \geq \varphi^{\sharp}\left(u_{m(n)-1}^{n}\right)$. Equation (5.23) has again three solutions given by $u_{m(n)-1}^{n}, u_{m(n)+1}^{n}$ and 
$\Psi\left(u_{m(n)-1}^{n}, u_{m(n)+1}^{n}\right)$. The value $\Psi(v, w) \in \mathbb{R} \backslash\{v, w\}$ is defined as the solution of

$$
\bar{a}(v, w)=\bar{a}(v, \Psi(v, w)) .
$$

By the same argument as in Case 1 we get that the limit equation (5.24) has also three solutions given by $v, w, \Psi(v, w)$. The situation $u_{-}(t)=v$, $u_{+}(t)=w$ fulfils the second part of (5.22), but $u_{-}(t)=v, u_{+}(t)=\Psi(v, w)$ in general not. However, the latter situation does not show up: we prove that for $u_{-}(t)=v, u_{+}(t)=\Psi(v, w)$ we get the contradiction

$$
D(t) \leq D^{*}(t)<D(t) .
$$

The first inequality in (5.25) was already shown in Lemma 5.12. To show the second one, we use (5.20), $v=u_{-}^{*}(t)$ and $w=u_{+}^{*}(t)$ (what is a consequence of $\left.u_{-}^{*}(t), u_{+}^{*}(t) \in\{v, w, \Psi(v, w)\}\right)$ and

$$
\bar{a}(v, w)=\bar{a}(v, \Psi(v, w))=\bar{a}(w, \Psi(v, w)),
$$

to find that $D^{*}(t)<D(t)$ is equivalent to

$$
-\bar{a}(w, \Psi(v, w))(\eta(\Psi(v, w))-\eta(w))+(q(\Psi(v, w))-q(w))>0 .
$$

The inequality (5.26) is finally correct, since the shock wave connecting $w$ to $\Psi(v, w)$ is not entropy consistent.

6. Numerical Experiments. In this section we will demonstrate the efficiency of Algorithm 3.1 numerically. We do this by calculating the so-called experimental order of convergence (EOC). For a given sequence of grid widths $h_{i}$ and corresponding numerical solutions $u_{h_{i}}$ we define the EOC by

$$
\mathrm{EOC}_{i}=\frac{\ln \left\|u_{h_{i}}-u\right\|_{L^{1}}-\ln \left\|u_{h_{i-1}}-u\right\|_{L^{1}}}{\ln h_{i}-\ln h_{i-1}} .
$$

By $u$ we denote here the $(\bar{\eta}, \varphi)$-solution with $\bar{\eta}(u)=\frac{1}{2} u^{2}$ and $\varphi$ to be fixed.

Furthermore, we will present an experiment showing the convergence of the trace values for non-Riemann initial data. The test was proposed in [3] and we compare our results with the results given there. We give also an example of an $(\bar{\eta}, \varphi)$-solution switching between a Laxian and an undercompressive phase transition, compare to Remark 2.4 (iii).

In the following experiments are done on equi-distant meshes with $N$ grid cells. For the numerical flux we use an upwind numerical flux except for Example 6.4, where a Lax-Friedrichs numerical flux is used.

EXAmPle 6.1 (Experimental Order of Convergence I). For Algorithm 3.1 we proved the convergence to an $(\bar{\eta}, \varphi)$-solution. The question of the rate remains open. Therefore we study the experimental order of convergence (EOC) for the scheme. We use the simple flux $f(u)=\frac{1}{3} u^{3}$ with $\varphi(u)=-\frac{3}{4} u$ as a kinetic relation. The entropy plays no role in this example. First we study the Riemann problem (1.1), (2.16) with $u_{L}=1$ and $u_{R}=-\frac{1}{2}$ on the domain $[-1,1]$ for different grids with $N$ cells. The $(\bar{\eta}, \varphi)$-solution is given by

$$
u(x, t)= \begin{cases}1 & \text { for } \frac{x}{t}<\frac{13}{48} \\ -\frac{3}{4} & \text { for } \frac{13}{48}<\frac{x}{t}<\frac{19}{48} \\ -\frac{1}{2} & \text { for } \frac{x}{t}>\frac{19}{48}\end{cases}
$$




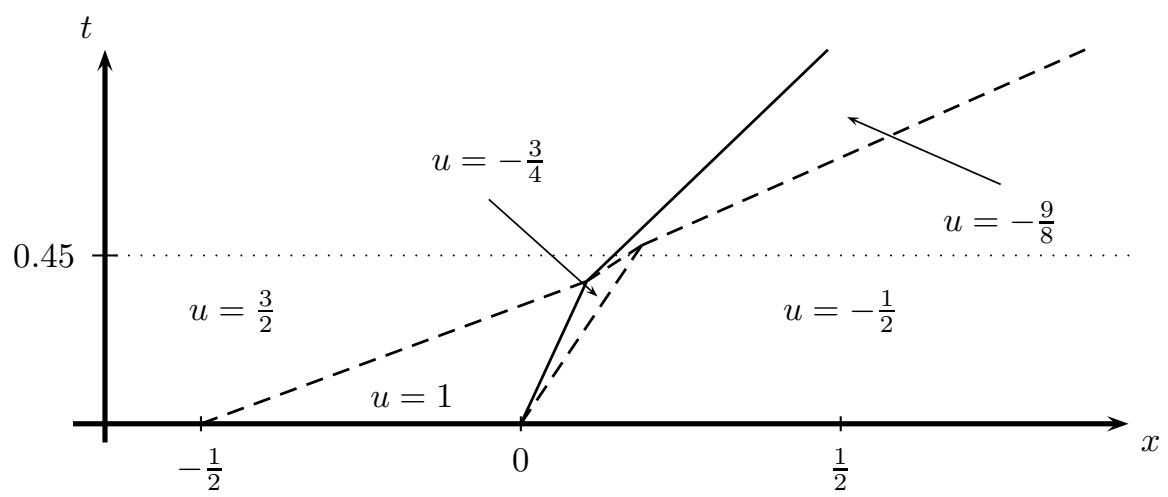

FIG. 6.1. Solution to initial conditions given in Example 6.2. Solid lines indicate phase boundaries and dashed line shock waves.

We measure the $L^{1}([-1,1])$-error at time $t=1$. The measured errors and resulting EOCs are listed in Table 6.1. We observe an approximately linear rate, which we can expect for Riemann initial data, since the undercompressive wave is exactly reproduced in this case.

\begin{tabular}{rcccc} 
& \multicolumn{2}{c}{ Example 6.1 } & \multicolumn{2}{c}{ Example 6.2} \\
$N$ & $L^{1}$-Error & EOC & $L^{1}$-Error & EOC \\
\hline 40 & 0.01567 & & 0.02791 & \\
80 & 0.00858 & 0.868 & 0.01592 & 0.810 \\
160 & 0.00449 & 0.934 & 0.00709 & 1.167 \\
320 & 0.00226 & 0.993 & 0.00389 & 0.864 \\
640 & 0.00113 & 1.000 & 0.00240 & 0.696 \\
1280 & 0.00056 & 1.000 & 0.00143 & 0.754 \\
2560 & 0.00028 & 1.000 & 0.00072 & 0.992 \\
5120 & 0.00014 & 1.000 & 0.00036 & 0.985 \\
\hline
\end{tabular}

Errors and EOC for the calculations done in Example 6.1 and 6.2 with different numbers of grid cells $N$.

ExAmPle 6.2 (Experimental Order of Convergence II). In contrast to Example 6.1 we use here initial data such that the phase boundary is not reproduced exactly. We achieve this by putting two discontinuities into the initial conditions, which evolve as single Laxian shock wave and phase boundary, and collide after some time. After the interaction the phase boundary contributes to the total error. The initial conditions are

$$
u_{0}(x)= \begin{cases}\frac{3}{2} & \text { for } x<-\frac{1}{2}, \\ 1 & \text { for }-\frac{1}{2}<x<0 \\ -\frac{1}{2} & \text { for } x>0 .\end{cases}
$$

The solution of this problem is illustrated in Figure 6.1. We measure the error in the $L^{1}([-1,1])$-norm at time $t=0.45$ (see Table 6.1). As in Example 6.1 we observe an EOC of 1 . 


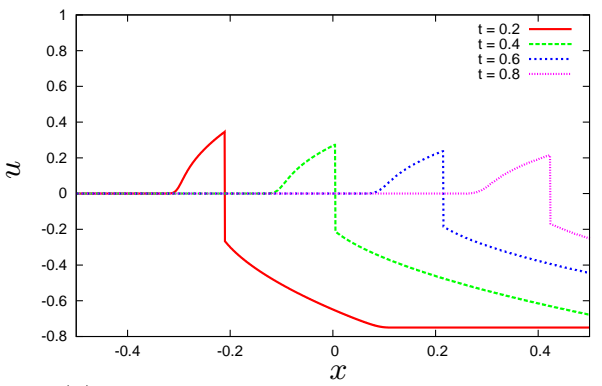

(a) Evolution of the discrete solution.

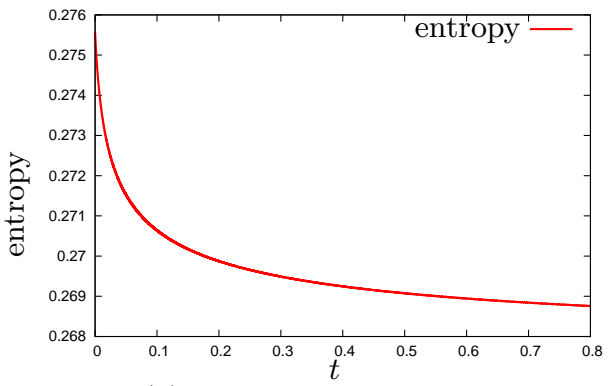

(b) Total Entropy decay.

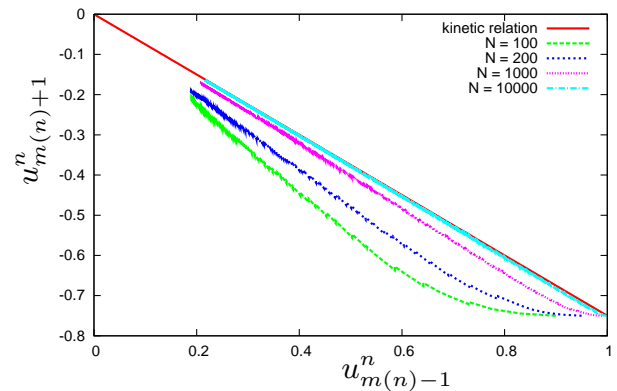

(c) Trace value history from $t=0$ to $t=0.8$.

Fig. 6.2. Non Riemann problem test case. The test is described in [3]. In 6.2(c) we mean by kinetic relation the graph $(u, \varphi(u))$.

ExAmple 6.3 (Non-Riemann Initial Conditions). To demonstrate the convergence rate of the trace values, we run Test $G$ from [3] on the interval $[-0.5,0.5]$. The results are visualized in Figure 6.2. The authors of [3] use $f(u)=u^{3}+u$ and $\varphi(u)=-\frac{3}{4} u$. As initial datum they choose

$$
u_{0}(x)= \begin{cases}0 & \text { for } x<-0.5 \\ 1+20(x+0.45) & \text { for } x \in[-0.5,-0.45] \\ -0.75 & \text { for } x>-0.45\end{cases}
$$

Figure 6.2(a) shows a numerical solution at different times on a grid with 5000 cells. Figure 6.2(b) shows the decay of the total entropy

$$
\int_{-\frac{1}{2}}^{\frac{1}{2}} \bar{\eta}(u(x, t)) \mathrm{d} x+\left.\int_{0}^{t} \bar{q}(u(x, s))\right|_{x=-\frac{1}{2}} ^{\frac{1}{2}} \mathrm{~d} s \quad \text { with } \bar{\eta}(u)=\frac{1}{2} u^{2}, \bar{q}(u)=\frac{3}{4} u^{4}+\frac{1}{2} u^{2} .
$$

in time for the same calculation. This confirms the statement of Theorem 5.8. In Figure 6.2(c) the trace values

$$
\left(u_{m(n)-1}^{n}, u_{m(n)+1}^{n}\right)_{n}
$$

are plotted. We observe convergence to the kinetic relation for $N \rightarrow \infty$, as already proved in Theorem 5.13. Calculating the EOC for the traces values gives: 0.1537, 0.5030 and 0.8765 . We expect a convergence rate of 0.5 . Compared to [3] we get the same qualitative behavior. 


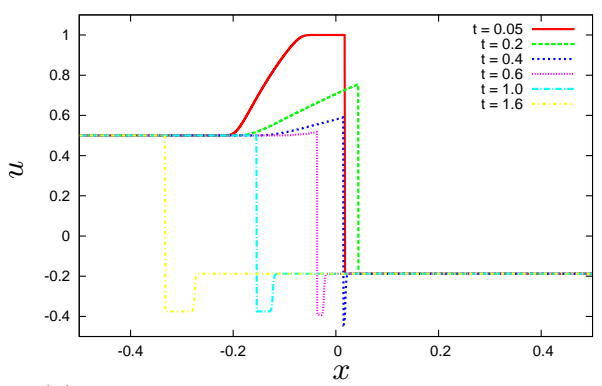

(a) Numerical solution at different times.

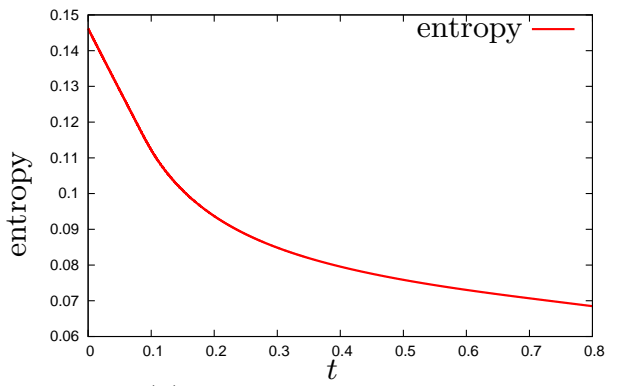

(b) Total Entropy decay.

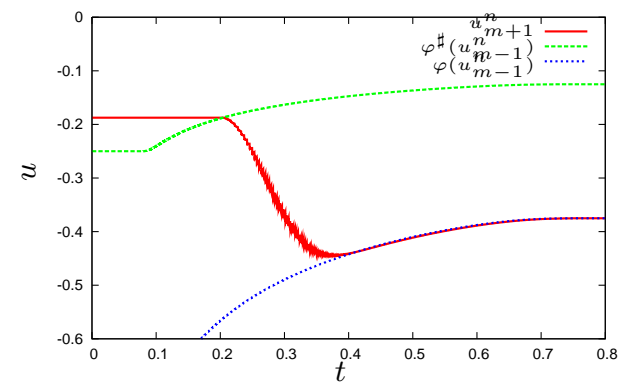

(c) The values $\varphi^{\sharp}\left(u_{m(n)-1}^{n}\right)$ and $u_{m(n)+1}^{n}$ over time.

FIG. 6.3. Initial value problem with a switch from Laxian to undercompressive phase boundaries. The switch takes place at approximately $t=0.2$.

ExAmPle 6.4 (Laxian and Undercompressive Phase Boundaries). In this example we give initial conditions for which the phase boundary switches from a Laxian to an undercompressive phase boundary. We used $f(u)=u^{3}-\frac{1}{2} u$ and $\varphi(u)=-\frac{3}{4} u$. The initial datum is given by

$$
u_{0}(x)= \begin{cases}\frac{1}{2} & \text { for } x<\frac{2}{5}, \\ 1 & \text { for } x \in\left[\frac{2}{5}, 0\right], \\ -\frac{3}{16} & \text { for } x>0 .\end{cases}
$$

For the discretization we used 5000 grid cells. The initial conditions are constructed such that the phase boundary propagates as a Laxian wave to the right. At approximately $t=0.1$ a rarefaction wave hits the phase boundary and makes it undercompressive for $t>0.2$. The undercompressive phase boundary now travels to the left. In Figure 6.3(c) the evolution of the traces values $\varphi^{\sharp}\left(u_{m(n)-1}^{n}\right)$ and $u_{m(n)+1}^{n}$ in time is plotted. The intersection point marks the switch from a Laxian to an undercompressive phase boundary. We refer to Remark 2.4 for the definition and (3.3) for the realization in the scheme.

Acknowledgments. The authors P. Engel and C. Rohde would like to thank the German Research Foundation (DFG) for financial support of the project within the Cluster of Excellence in Simulation Technology (EXC 310/1) at the University of Stuttgart.

\section{REFERENCES}


[1] Rohan Abeyaratne and James K. Knowles, Kinetic relations and the propagation of phase boundaries in solids, Arch. Rational Mech. Anal., 114 (1991), pp. 119-154.

[2] A. L. Bertozzi, A. Münch, And M. Shearer, Undercompressive shocks in thin film flows, Phys. D, 134 (1999), pp. 431-464.

[3] Benjamin Boutin, Christophe Chalons, Frédéric Lagoutière, and Philippe G. LeFloch, Convergent and conservative schemes for nonclassical solutions based on kinetic relations. I, Interfaces Free Bound., 10 (2008), pp. 399-421.

[4] Christophe Chalons, Frédéric Coquel, Patrick Engel, and Christian Rohde, Fast relaxation solvers for hyperbolic-elliptic phase transition problems, SIAM Journal on Scientific Computing, 34 (2012).

[5] C. Chalons and P. G. LeFloch, Computing undercompressive waves with the random choice scheme. Nonclassical shock waves, Interfaces Free Bound., 5 (2003), pp. 129-158.

[6] Rinaldo M. Colombo and Andrea Corli, Sonic and kinetic phase transitions with applications to Chapman-Jouguet deflagrations, Math. Methods Appl. Sci., 27 (2004), pp. 843-864.

[7] Rinaldo M. Colombo and Massimiliano D. Rosini, Pedestrian flows and non-classical shocks, Math. Methods Appl. Sci., 28 (2005), pp. 1553-1567.

[8] Andrea Corli and Christian Rohde, Singular limits for a parabolic-elliptic regularization of scalar conservation laws, J. Differential Eq., 253 (2012), pp. 1399-1421.

[9] Constantine M. Dafermos, Hyperbolic conservation laws in continuum physics, vol. 325 of Grundlehren der Mathematischen Wissenschaften [Fundamental Principles of Mathematical Sciences], Springer-Verlag, Berlin, third ed., 2010.

[10] Volker Elling, A Lax-Wendroff type theorem for unstructured quasi-uniform grids, Math. Comp., 76 (2007), pp. 251-272.

[11] Ami Harten, High resolution schemes for hyperbolic conservation laws, J. Comput. Phys., 49 (1983), pp. 357-393.

[12] Brian T. Hayes and Philippe G. LeFloch, Non-classical shocks and kinetic relations: scalar conservation laws, Arch. Rational Mech. Anal., 139 (1997), pp. 1-56.

[13] — Nonclassical shocks and kinetic relations: finite difference schemes, SIAM J. Numer. Anal., 35 (1998), pp. 2169-2194.

[14] Thomas Y. Hou, Phoebus Rosakis, and Philippe LeFloch, A level-set approach to the computation of twinning and phase-transition dynamics, J. Comput. Phys., 150 (1999), pp. 302-331.

[15] Frederike Kissling And Christian Rohde, The computation of nonclassical shock waves with a heterogeneous multiscale method, Netw. Heterog. Media, 5 (2010), pp. 661-674.

[16] A. Kluwick, E.A. Cox, And S. Scheichl, Non-classical kinematic shocks in suspensions of particles in fluids, Acta Mech., 144 (2000), pp. 197-210.

[17] Dietmar Kröner, Numerical schemes for conservation laws, Wiley-Teubner Series Advances in Numerical Mathematics, John Wiley \& Sons Ltd., Chichester, 1997.

[18] Philippe LeFloch, Propagating phase boundaries: formulation of the problem and existence via the Glimm method, Arch. Rational Mech. Anal., 123 (1993), pp. 153-197.

[19] Philippe G. LeFloch, Hyperbolic systems of conservation laws, Lectures in Mathematics ETH Zürich, Birkhäuser Verlag, Basel, 2002. The theory of classical and nonclassical shock waves.

[20] P. G. LeFloch, J. M. Mercier, And C. Rohde, Fully discrete, entropy conservative schemes of arbitrary order, SIAM J. Numer. Anal., 40 (2002), pp. 1968-1992.

[21] Philippe G. LeFloch and Majid Mohammadian, Why many theories of shock waves are necessary: kinetic functions, equivalent equations, and fourth-order models, J. Comput. Phys., 227 (2008), pp. 4162-4189.

[22] Philippe G. LeFloch and Christian Rohde, High-order schemes, entropy inequalities, and nonclassical shocks, SIAM J. Numer. Anal., 37 (2000), pp. 2023-2060.

[23] C. Merkle and C. Rohde, Computation of dynamical phase transitions in solids, Appl. Numer. Math., 56 (2006), pp. 1450-1463.

[24] Christian Merkle and Christian Rohde, The sharp-interface approach for fluids with phase change: Riemann problems and ghost fluid techniques, M2AN Math. Model. Numer. Anal., 41 (2007), pp. 1089-1123.

[25] Vincent Perrier, A conservative method for the simulation of the isothermal Euler system with the van-der-Waals equation of state, J. Sci. Comput., 48 (2011), pp. 296-303. 
[26] Christian Rohde, Scalar conservation laws with mixed local and nonlocal diffusion-dispersion terms, SIAM J. Math. Anal., 37 (2005), pp. 103-129.

[27] Richard SANDERs, The moving grid method for nonlinear hyperbolic conservation laws, SIAM J. Numer. Anal., 22 (1985), pp. 713-728.

[28] L. TRUSKinOvSKY, Kinks versus shocks, in Shock induced transitions and phase structures in general media, vol. 52 of IMA Vol. Math. Appl., Springer, New York, 1993, pp. 185-229.

[29] C. J. van Duijn, L. A. Peletier, and I. S. Pop, A new class of entropy solutions of the Buckley-Leverett equation, SIAM J. Math. Anal., 39 (2007), pp. 507-536.

[30] Xiaoguang Zhong, Thomas Y. Hou, and Philippe G. LeFloch, Computational methods for propagating phase boundaries, J. Comput. Phys., 124 (1996), pp. 192-216. 